\title{
Viscous coupling of shear-free turbulence across nearly flat fluid interfaces
}

\section{Article}

Published Version

Hunt, J. C. R., Stretch, D. D. and Belcher, S. E. (2011)

Viscous coupling of shear-free turbulence across nearly flat fluid interfaces. Journal Of Fluid Mechanics, 671. pp. 96-120. ISSN 0022-1120 doi: https://doi.org/10.1017/s0022112010005525 Available at https://centaur.reading.ac.uk/26970/

It is advisable to refer to the publisher's version if you intend to cite from the work. See Guidance on citing.

Published version at: http://dx.doi.org/10.1017/s0022112010005525

To link to this article DOI: http://dx.doi.org/10.1017/s0022112010005525

Publisher: Cambridge University Press

All outputs in CentAUR are protected by Intellectual Property Rights law, including copyright law. Copyright and IPR is retained by the creators or other copyright holders. Terms and conditions for use of this material are defined in the End User Agreement.

\section{www.reading.ac.uk/centaur}

\section{CentAUR}

Central Archive at the University of Reading 
Reading's research outputs online 


\title{
Viscous coupling of shear-free turbulence across nearly flat fluid interfaces
}

\author{
J. C. R. HUNT T ${ }^{1,2} \dagger$, D. D. STRETCH AND S. E. BELCHER \\ ${ }^{1}$ Department of Earth Sciences, University College London, London WC1E 6BT, UK \\ ${ }^{2}$ J. M. Burgers Centre, Delft University of Technology, 2628 CD Delft, The Netherlands \\ ${ }^{3}$ School of Civil Engineering, University of KwaZulu Natal, Durban 4041, South Africa \\ ${ }^{4}$ Department of Meteorology, University of Reading, Reading RG6 6BB, UK
}

(Received 12 August 2009; revised 27 May 2010; accepted 15 October 2010)

The interactions between shear-free turbulence in two regions (denoted as + and - ) on either side of a nearly flat horizontal interface are shown here to be controlled by several mechanisms, which depend on the magnitudes of the ratios of the densities, $\rho_{+} / \rho_{-}$, and kinematic viscosities of the fluids, $\mu_{+} / \mu_{-}$, and the root mean square (r.m.s.) velocities of the turbulence, $u_{0+} / u_{0-}$, above and below the interface. This study focuses on gas-liquid interfaces so that $\rho_{+} / \rho_{-} \ll 1$ and also on where turbulence is generated either above or below the interface so that $u_{0+} / u_{0-}$ is either very large or very small. It is assumed that vertical buoyancy forces across the interface are much larger than internal forces so that the interface is nearly flat, and coupling between turbulence on either side of the interface is determined by viscous stresses. A formal linearized rapid-distortion analysis with viscous effects is developed by extending the previous study by Hunt \& Graham (J. Fluid Mech., vol. 84, 1978, pp. 209-235) of shear-free turbulence near rigid plane boundaries. The physical processes accounted for in our model include both the blocking effect of the interface on normal components of the turbulence and the viscous coupling of the horizontal field across thin interfacial viscous boundary layers. The horizontal divergence in the perturbation velocity field in the viscous layer drives weak inviscid irrotational velocity fluctuations outside the viscous boundary layers in a mechanism analogous to Ekman pumping. The analysis shows the following. (i) The blocking effects are similar to those near rigid boundaries on each side of the interface, but through the action of the thin viscous layers above and below the interface, the horizontal and vertical velocity components differ from those near a rigid surface and are correlated or anti-correlated respectively. (ii) Because of the growth of the viscous layers on either side of the interface, the ratio $u_{I} / u_{0}$, where $u_{I}$ is the r.m.s. of the interfacial velocity fluctuations and $u_{0}$ the r.m.s. of the homogeneous turbulence far from the interface, does not vary with time. If the turbulence is driven in the lower layer with $\rho_{+} / \rho_{-} \ll 1$ and $u_{0+} / u_{0-} \ll 1$, then $u_{I} / u_{0_{-}} \sim 1$ when $\operatorname{Re}\left(=u_{0-} L_{-} / \nu_{-}\right) \gg 1$ and $R=\left(\rho_{-} / \rho_{+}\right)\left(v_{-} / v_{+}\right)^{1 / 2} \gg 1$. If the turbulence is driven in the upper layer with $\rho_{+} / \rho_{-} \ll 1$ and $u_{0+} / u_{0-} \gg 1$, then $u_{I} / u_{0+} \sim 1 /(1+R)$. (iii) Nonlinear effects become significant over periods greater than Lagrangian time scales. When turbulence is generated in the lower layer, and the Reynolds number is high enough, motions in the upper viscous layer are turbulent. The horizontal vorticity tends to decrease, and the vertical vorticity of the eddies dominates their asymptotic structure. When turbulence is generated in the upper layer, and the Reynolds number is less than about $10^{6}-10^{7}$, the fluctuations in the 
viscous layer do not become turbulent. Nonlinear processes at the interface increase the ratio $u_{I} / u_{0+}$ for sheared or shear-free turbulence in the gas above its linear value of $u_{I} / u_{0+} \sim 1 /(1+R)$ to $\left(\rho_{+} / \rho_{-}\right)^{1 / 2} \sim 1 / 30$ for air-water interfaces. This estimate agrees with the direct numerical simulation results from Lombardi, De Angelis \& Bannerjee (Phys. Fluids, vol. 8, no. 6, 1996, pp. 1643-1665). Because the linear viscous-inertial coupling mechanism is still significant, the eddy motions on either side of the interface have a similar horizontal structure, although their vertical structure differs.

Key words: air/sea interactions, turbulence theory

\section{Introduction}

We consider two fluids separated by an interface. Each fluid is in turbulent motion, and there is no mean flow. The literature (e.g. Brutsaert \& Jirka 1984) indicates the wide range of flow phenomena involving interface motion and the coupling between the adjacent velocity and scalar fields. The nature of the coupling is characterized by the ratios of the densities, $\rho_{+} / \rho_{-}$, the viscosities, $\mu_{+} / \mu_{-}$, and the root mean square (r.m.s.) values of velocity fields far from the interface, $u_{+}^{\prime} / u_{-}^{\prime}$ (here + and - denote properties above and below the interface), and by the interface Froude number, which is defined by

$$
F_{I}=\frac{u_{0}^{2}}{g^{\prime} L},
$$

where the reduced gravity is

$$
g^{\prime}=g \frac{\left(\rho_{-}-\rho_{+}\right)}{0.5\left(\rho_{-}+\rho_{+}\right)}
$$

and $u_{0}$ and $L$ are the velocity and length scales of the forcing turbulence (either above or below the interface). The Reynolds numbers are taken to be large so that $R e_{+}=u_{+}^{\prime} L / v_{+} \gg 1$ and similarly $R e_{-} \gg 1$.

There are four broad classes of interface motion that are qualitatively different.

(i) Turbulence forced in the lower fluid: when $\rho_{+} / \rho_{-} \ll 1$ and $F_{I} \ll 1$ and if initially $u_{-}^{\prime} \gg u_{+}^{\prime}$, turbulence within the lower region is blocked by the interface and drives fluctuations in the upper region by viscous action

(ii) As $\rho_{+} / \rho_{-}$and $F_{I}$ increase, turbulence in the lower region produces moving distortions of the lower interface which produce larger motions in the upper region by viscous stresses (Brocchini \& Peregrine 2001; Fulgosi et al. 2003; Teixeira \& Belcher 2006; Lin et al. 2008).

(iii) Turbulence forced in the upper fluid: when $\rho_{+} / \rho_{-} \ll 1$ and $F_{I} \ll 1$ and if initially $u_{-}^{\prime} \ll u_{+}^{\prime}$, turbulence in the upper region is blocked by the interface and drives fluctuations in the lower region by viscous action

(iv) For larger values of $\rho_{+} / \rho_{-}<1$ and for $F_{I} \sim 1$ turbulence in the upper region distorts the interface by driving surface waves (e.g. Belcher \& Hunt 1998) and triggers Kelvin-Helmholtz instabilities so that motions in the lower region are driven by shear stresses and normal motions of the interface (Teixeira \& Belcher 2006). The waves themselves then further distort the turbulence (Teixeira \& Belcher 2002).

In regime (i) the flow is driven by turbulent fluctuations in the lower layer (e.g. by rain drops impinging on the interface; Takagaki \& Komori 2007), which then, through viscous coupling across the flat interface, drive motions in the upper layer. 
The imposed turbulence is either isotropic or, if anisotropic, statistically orientated symmetrically (i.e. normal or perpendicular) with respect to the interface so that no mean flow is generated (Nagata et al. 2006). This coupling also affects transport across the interface, especially when there is also a turbulent flow in the upper layer (Komori, Nagaosa \& Murakami 1993). Magnaudet (2003) has shown theoretically why the linear inviscid blocking mechanism of Hunt \& Graham (1978) controls the normal fluctuation of the interface over long periods even when nonlinear effects become generally significant. But he did not consider the significant changes in eddy structure that develop over large times. These distortions of the surface and of the eddy structure near the surface are greater in regime (ii): when $\rho_{+} / \rho_{-} \approx 1, F_{I} \geqslant 1$ and $R e \gg 1$ motions in region + driven by the lower layer are largely irrotational and are similar to those outside other non-turbulent-turbulent interfaces (e.g. the edge of a wake; Phillips 1955; Carruthers \& Hunt 1986).

Regime (iii) occurs when turbulent motions in the upper layer drive motions in the lower layer, such as gas convecting over warmer liquid. Then the gas motions drive, through viscous shear stresses at the interface, eddying in the lower layer. Depending on the relative thermal diffusivities $\left(K_{+} / K_{-}\right)$, this eddying controls the heat transfer into the upper layer and affects the buoyancy forces, the latter of which in turn determines the strength of turbulence in the upper layer and the 'plume' or unsteady 'puff-like' structure of the large eddies (Hunt et al. 2003).

In regime (iv), when $F_{I}$ is larger, the interface is substantially deflected, and strong interfacial wave motions are generated, which eventually break down into a layer of spray droplets and foam. The coupling of the turbulence is first increased by the surface deflections but eventually decreases as the foam layer forms and isolates the two flow fields.

Lombardi et al. (1996) considered turbulent pressure-driven flows in a domain half filled with gas and half filled with liquid, with the interface constrained to be flat but across which the horizontal velocity and shear stress were continuous. They performed simulations and investigated the turbulence structure in each case for various values of the ratio of the fluid densities, namely $\rho_{-} / \rho_{+}=1,100,900$ (the latter corresponds approximately to an air-water interface, where $\nu_{-} / \nu_{+}=1 / 10$ and $\mu_{+} / \mu_{-} \sim 10^{-2}$ ). Significant differences were found between the turbulent motions above and below the interface. In particular, they found that for air-water flow, the turbulent motions in the upper gas layer $(+)$ have a similar streak-like structure to a wall-bounded turbulent shear flow. But the turbulence in the lower liquid layer (-) has a different structure because it is driven both by the mean shear and by horizontal fluctuations of the interface. Although the shear produces a similar streak-like structure, there are qualitative and quantitative differences with the upper layer turbulence. For example, the turbulent intensities and the Reynolds shear stress near the interface increase with $\rho_{-} / \rho_{+}$, and the horizontal velocity fluctuations on the liquid side develop a peak at the interface when $\rho_{-} / \rho_{+}=900$ (but not for the case $\rho_{-} / \rho_{+}=100$, where these fluctuations are damped by the viscous stresses at the interface, and they peak below the interface). Turbulent kinetic energy production and dissipation rates near the interface are about the same in the liquid as in the gas. They also examined the instantaneous flow structure near the interface, using a conditional-sampling quadrant analysis of the Reynolds stress contributions and visualization of quasi-streamwise vortices. In the gas, they found that 'sweep' (or fourth-quadrant) events occur with high probability over regions with large interfacial stress, while 'ejection' (secondquadrant) events occur over low-stress regions. This behaviour is similar to that in turbulent boundary layers over a rigid surface (e.g. Kim, Moin \& Moser 1987; 
Guezennec, Stretch \& Kim 1990). In the liquid motions, however, this correlation of sweeps and ejections with the local instantaneous shear stress was not observed. They concluded that turbulence in the liquid is relatively more 'inactive'. The numerical simulations provide valuable data but do not explain, for example, why the structure of the turbulent motions in the liquid changes as the density ratio $\rho_{-} / \rho_{+}$increases. It is also interesting to consider how turbulence in the upper layer gas is affected by turbulence in the liquid layer.

Here we investigate these processes by using rapid-distortion theory. The mechanisms are analysed by calculating the 'rapid' response of initially homogeneous shear-free turbulence to the presence of the interface, so as to calculate how the velocity fluctuations are coupled dynamically and kinematically across the interface.

Rapid-distortion theory (referred to here as RDT) is formally valid only over a short period following a sudden change of the boundary conditions or the mean flow when nonlinear interactions between eddies are relatively small. However, in many distorted flows, turbulence statistics such as ratios of moments tend to vary slowly with time according to RDT predictions and, as measurements and simulations demonstrate, also over longer times of order $T_{L} \sim L_{0} / U_{0}$. Hunt \& Graham (1978), Townsend (1976), Hunt \& Carruthers (1990) and Magnaudet (2003) developed theoretical arguments to explain why the nonlinear effects are weak near interfaces. The present analysis applies to initially homogeneous shear-free turbulence near nearly flat fluid interfaces by firstly considering thin viscous layers above and below the interface and secondly using these solutions to provide coupling of the fluctuations in the source layers. The Kelvin-Helmholtz instabilities and waves are negligible for the parameter regime considered where $F_{I} \ll 1$. The results of the analysis also indicate some of the key coupling/transfer processes for turbulent shear flows.

\section{Mathematical formulation of the problem}

Suppose that initially a fluid of density $\rho_{-}$lies in $z<0$ below a fluid of density $\rho_{+}$in $z>0$. Then at time $t=0$ the fluid in the lower region $z<0$ (region - ) is set into homogeneous turbulent motion, $u_{-}^{(H)}$, with velocity scale $u_{0}$, length scale $L_{0}$ and Reynolds number $R e=u_{0} L_{0} / v \gg 1$. There is no mean shear so that (for small times) $\bar{u}=0$. When the turbulence is generated the interface transmits a 'rapid' distortion of the turbulence in the lower fluid layer. Pressure fluctuations generated in the two layers match at the interface, leading to the deformation of the interface, with an amplitude that depends on the Froude number $F_{L}$ (Fernando \& Hunt 1997). In this paper we focus on flows in which $F_{L} \ll 1$ and the interface is flat (the dynamical condition for which is given in the Appendix) so that the vertical velocity fluctuations are zero at $z=0$. The flow in the upper layer is then driven by the continuity of horizontal viscous stresses at the interface. This coupling is analysed here using RDT, when the nonlinear interactions are weak compared with the effects of the boundary conditions. The analysis is then valid for a time $t$ that is smaller than an eddy turnover time $L_{0} / u_{0}$, and hence it is valid for longest times for the largest eddies, which are also the most energetic (Hunt \& Graham 1978, hereafter HG).

For short times when the nonlinear interactions between turbulent eddies are small, the response to the sudden imposition of the interface can be described by the linearized Navier-Stokes equations (in both layers), together with the continuity equation,

$$
\frac{\partial \boldsymbol{u}}{\partial t}=-\frac{1}{\rho} \nabla p+v \nabla^{2} \boldsymbol{u}, \quad \nabla \cdot \boldsymbol{u}=0 .
$$


When the Reynolds number is large, $R e \gg 1$, the viscous effects are important only in narrow boundary layers on either side of the interface; the bulk of the flow is inviscid.

The mathematical theory that follows focuses on regimes (i) and (iii). Similar methods of solution are used for each regime, although the solutions differ in important respects. Since the governing equations and boundary conditions are linear, linear superposition can be used to study the combined effects of turbulence generated in the upper and lower layers simultaneously (e.g. Wu \& Fernando 1999).

\section{Mathematical formulation of the problem, mechanisms and scaling}

\subsection{Turbulence driven in the lower layer: regime ( $i$ )}

First consider the evolution of turbulence on either side of the interface when initially there is homogenous turbulence, $\boldsymbol{u}_{-}^{(H)}$, in the lower fluid $z<0$ only. Its r.m.s. velocity is $u_{0-}$ abbreviated to $u_{0}$. The effect of the sudden imposition of the interface is to distort the turbulence into a four-layer structure, which is shown schematically in figure 1. As discussed below, the solution to the linear equations (2.1) are then written as three components, which are also developed in $\mathrm{HG}$, describing homogeneous $(H)$, source $(S)$, and viscous $(V)$ layers. Here these components are augmented by a component arising from the viscous layer-source layer interaction denoted $(V, S)$ and also by coupling across the interface. Hence the solution for the velocity, $\boldsymbol{u}=(u, v, w)$, is written as

$$
\left.\begin{array}{ll}
\boldsymbol{u}=\boldsymbol{u}_{-}=\boldsymbol{u}_{-}^{(H)}+\boldsymbol{u}_{-}^{(S)}+\boldsymbol{u}_{-}^{(V)}+\boldsymbol{u}_{-}^{(V, S)}, & z<0, \\
\boldsymbol{u}=\boldsymbol{u}_{+}=\boldsymbol{u}_{+}^{(V)}+\boldsymbol{u}_{+}^{(V, S)}, & z>0 .
\end{array}\right\}
$$

The initial conditions at $t=0+$ are as follows:

$$
\boldsymbol{u}_{-}=\boldsymbol{u}_{-}^{(H)}, \quad z<0 ; \quad \boldsymbol{u}_{+}=0, \quad z>0 .
$$

The boundary conditions are

$$
\left.\begin{array}{lll}
\boldsymbol{u}_{-} \rightarrow \boldsymbol{u}_{-}^{(H)} & \text { as } & z \rightarrow-\infty \\
\boldsymbol{u}_{+} \rightarrow 0 & \text { as } & z \rightarrow \infty
\end{array}\right\}
$$

At the interface, the velocity and stress are continuous:

$$
\left.\begin{array}{l}
\left(u_{-}, v_{-}\right)=\left(u_{+}, v_{+}\right), \quad w_{-}=w_{+}=0, \\
\mu_{-}\left(\frac{\partial u_{-}}{\partial z}, \frac{\partial v_{-}}{\partial z}\right)=\mu_{+}\left(\frac{\partial u_{+}}{\partial z}, \frac{\partial v_{+}}{\partial z}\right),
\end{array}\right\} \quad \text { at } z=0
$$

\subsection{Source layer $S_{-}$}

The flat interface is imposed at $t=0$, and it remains flat; then the dynamic condition for the interface is satisfied (see the Appendix). The kinematic boundary condition that the vertical velocity is zero (i.e. $w_{-}=0$ ) is satisfied at $z=0$ by the addition of a new velocity field $\boldsymbol{u}_{-}^{(S)}$ such that at the surface $w_{-}^{(S)}(z=0)=-w_{-}^{(H)}(z=0)$. The $\boldsymbol{u}_{-}^{(S)}$ velocity field may therefore be thought of as generated by a collection of "virtual sources' on the interface that lead to a source layer, with a depth of order $L_{0}$. The linearized vorticity equation, derived from (2.1), reduces (as in $\mathrm{HG}$ ) to

$$
\frac{\partial \omega_{i-}}{\partial t} \approx v_{-} \nabla^{2} \omega_{i-}
$$


(a)

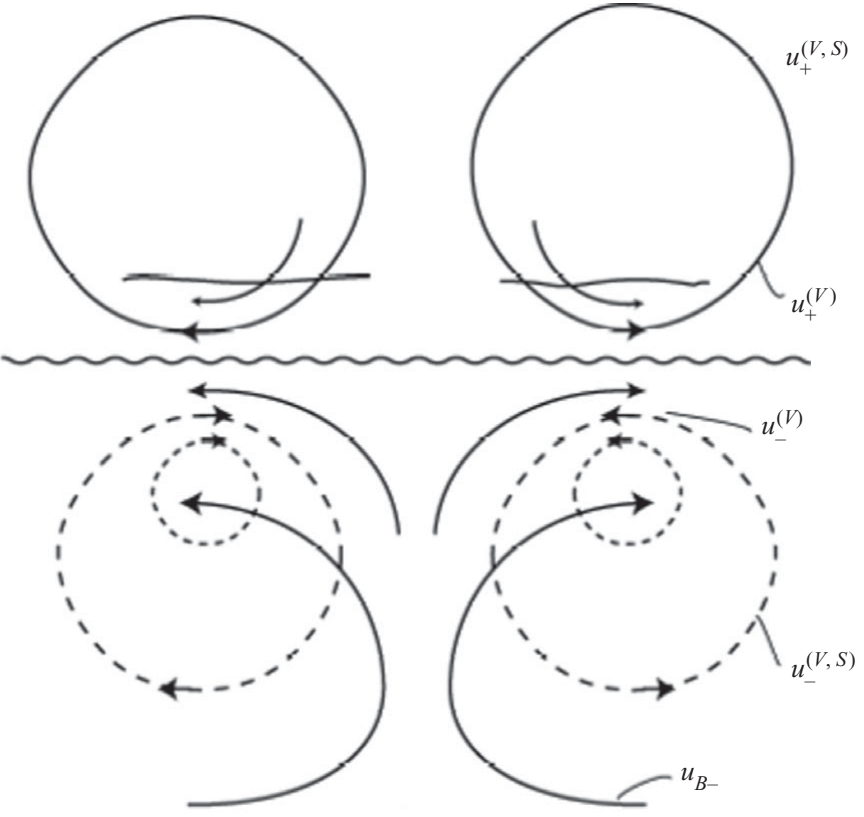

(b)

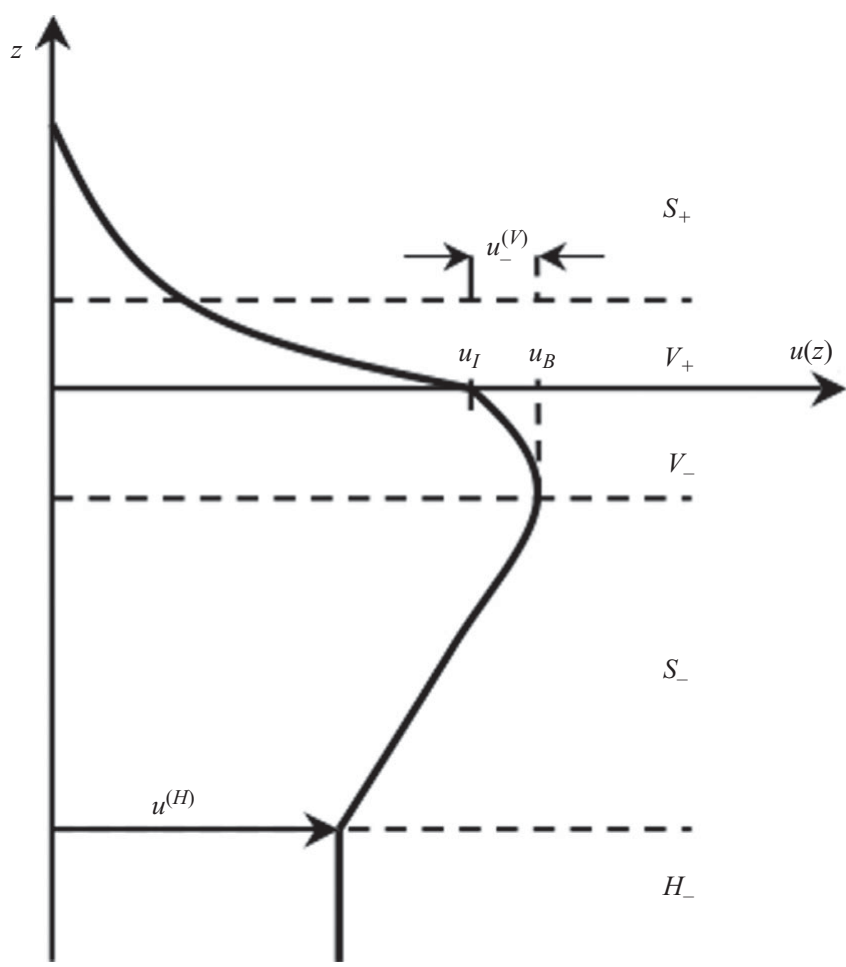

FIGURE 1 . Schematic diagrams of $(a)$ the eddy structure and $(b)$ the velocity profile for regime (i) when turbulence is forced in the lower fluid and when coupling across the interface produces circulations of the opposite sign in the upper fluid. The dashed lines in $(a)$ denote the contribution of the viscous perturbation in the lower layer. 
This shows that, for $R e \gg 1$, any vorticity generated by the introduction of the interface at $t=0$ is confined to a viscous boundary layer of thickness $\delta_{-}$below the interface so that for $-L_{0} \leqslant z<-\delta_{-}, \partial \omega_{i-} / \partial t \approx 0$. Therefore in the source layer $u_{-}^{(S)}$ is irrotational (HG).

An important feature of the flow distortion in the source layer is that the irrotational contributions in the source layer lead to the surface convergence and divergence $\partial u / \partial x+\partial v / \partial y$ being initially larger and more organized than in $H_{-}$. But this decreases eventually with time. This may explain why surface waves can be either generated or suppressed by the turbulence under the surface (Rozenberg, Matusov \& Mellvile 1998).

Since the boundary has the effect of blocking the velocity fluctuations, it is convenient in the later analysis to define the 'blocked' velocity field at the interface as

$$
\boldsymbol{u}_{B}=\boldsymbol{u}_{-}^{(H)}(z \rightarrow 0)+\boldsymbol{u}_{-}^{(S)}(z \rightarrow 0) .
$$

Hence $\boldsymbol{u}_{B}$ has zero vertical velocity by construction of $\boldsymbol{u}_{-}^{(S)}$. Notice that, since both $\boldsymbol{u}_{-}^{(H)}$ and $\boldsymbol{u}_{-}^{(S)}$ are of order $u_{0}$, the blocking velocity in the source layer, $\boldsymbol{u}_{B}$, is also of the order of the forcing turbulence, $u_{0}$.

\subsection{Viscous layers $V_{-}$and $V_{+}$}

The presence of the flat interface initiates the growth on either side of the interface of thin viscous boundary layers, with thicknesses

$$
\delta_{ \pm} \sim\left(v_{ \pm} t\right)^{1 / 2}\left(t<T_{0} \sim L_{0} / u_{0}\right) .
$$

Continuity of the horizontal viscous shear stress $\tau$ across the interface mediates coupling of the horizontal velocity field across the interface.

The strength of coupling across the interface can be understood with scaling arguments. On referring to figure $1(b)$, we see that the velocity at the top of the source layer $S_{-}$is $\boldsymbol{u}_{B}$ and the velocity at the interface is $\boldsymbol{u}_{I}$. The change in velocity across the viscous layer $V_{-}$is $\boldsymbol{u}_{-}^{(V)}$. The motions in the upper source layer $S_{+}$are small, and therefore the change in velocity across the upper viscous layer $V_{+}$is $\boldsymbol{u}_{I}$. Scaling estimates for $u_{I}$ and $\boldsymbol{u}_{-}^{(V)}$ can then be found by matching the fluid velocity and stress at the interface:

$$
\begin{gathered}
\boldsymbol{u}_{I}=\boldsymbol{u}_{B}+\boldsymbol{u}_{-}^{(V)}, \\
\mu_{+} \frac{\partial \boldsymbol{u}_{+}}{\partial z}=\mu_{-} \frac{\partial \boldsymbol{u}_{-}}{\partial z} .
\end{gathered}
$$

The velocity gradients can be estimated to be $\partial \boldsymbol{u}_{+} / \partial z \sim \boldsymbol{u}_{I} / \delta_{+}$and $\partial \boldsymbol{u}_{-} / \partial z \sim \boldsymbol{u}_{-}^{(V)} / \delta_{-}$ - if the very small gradient in $\boldsymbol{u}_{B}$ is ignored (it is retained in the precise analysis in $\S 4)$. Combination of these estimates then yields

$$
\begin{gathered}
\boldsymbol{u}_{I} \sim \boldsymbol{u}_{B} /\left(1+R^{-1}\right), \\
\boldsymbol{u}_{-}^{(V)} \sim-\boldsymbol{u}_{B} /(1+R),
\end{gathered}
$$

where the coupling parameter is

$$
R=\frac{\rho_{-}}{\rho_{+}}\left(\frac{v_{-}}{v_{+}}\right)^{1 / 2} \gg 1 .
$$


These scaling estimates have several noteworthy features. Firstly, since there is no net horizontal force acting across the interface, conservation of momentum (given in $(4.6 a)$ ) integrated from below the lower viscous layer to above the upper viscous layer shows that the depth-integrated transport in the upper and lower layers balance,

$$
\int_{-\delta_{-}}^{0} \rho_{-} \boldsymbol{u}_{-}^{(V)} \mathrm{d} z+\int_{0}^{\delta_{+}} \rho_{+} \boldsymbol{u}_{+}^{(V)} \mathrm{d} z=0,
$$

so that $\rho_{-} \boldsymbol{u}_{-}^{(V)} \delta_{-} \sim-\rho_{+} \boldsymbol{u}_{I} \delta_{+}$, which implies that $\boldsymbol{u}_{I} \sim-R \boldsymbol{u}_{-}^{(V)}$ (confirming $(3.4 a, b)$ ). Secondly, for this regime, $(3.4 a)$ implies that the interfacial velocity, $\boldsymbol{u}_{I}$, is of the same order as the blocking velocity, $\boldsymbol{u}_{B}$, and hence the imposed turbulence. Notice that $\boldsymbol{u}_{I}$ is independent of time because the depths of both viscous layers increase at the same rate. Hence, although the analysis is formally valid only for short times, we might expect this result to remain valid for longer times. Thirdly, (3.3c) and (3.4b) show that the maximum horizontal velocity occurs at the top of the lower source layer $S_{-}$, below the interface and the lower viscous layer $V_{-}$(as sketched in figure $1 b$ ). Fourthly, when $R$ is large, the change in velocity over the lower viscous layer, $\boldsymbol{u}_{-}^{(V)}$, is small. Hence the horizontal components of vorticity are small in the lower viscous layer, $V_{-}$, below the interface. As the viscous layer deepens over longer times, of order $t \sim\left(L_{0} / u_{0}\right) R e^{1 / 2}$, the viscous layer becomes $\delta_{-} \sim L_{o}$, and the horizontal vorticity is reduced over the whole source layer. The vertical component of the vorticity, in contrast, is unaffected by the viscous layer. Then, as the numerical solution of Tsai (1998) and some of the photographs of Brocchini \& Peregrine (2001) nicely demonstrate, in decaying or remotely generated turbulence the large-scale near-surface flow is dominated by vortices normal to the interface.

\subsection{Vertical motions induced by the viscous layers and $(V, S)$ contributions}

The velocity fields in $V_{-}$and $V_{+}$have, in general, a non-zero horizontal divergence. Hence, there is a vertical velocity at the outer edge of $V_{-}$that drives a perturbation in the source layer with irrotational velocity fluctuations denoted by $\boldsymbol{u}_{-}^{(V, S)}$. Similarly, vertical motions are produced in the upper layer at the outer edge of $V_{+}$, which leads to an irrotational source layer, with depth of order $L_{0}$ and with velocity $\boldsymbol{u}_{+}^{(V, S)}$, in the upper fluid. This mechanism of coupling with the upper fluid is more efficient than purely viscous coupling, which can only diffuse out to the viscous length $\delta_{+}$. It is similar to Ekman pumping in large-scale atmosphere-ocean dynamics (see e.g. Gill 1982, p. 326).

By continuity, the largest eddies lead to a vertical velocity in the upper source layer, $S_{+}$, of order

$$
w_{+}^{(V, S)} \sim\left(\delta_{+} / L_{o}\right) u_{I} \sim R e^{-1 / 2}\left(\frac{u_{0} t}{L_{0}}\right)^{1 / 2} u_{0},
$$

which is much smaller than $u_{0}$ for $t \leqslant T_{0}=L_{0} / U_{0}$. Equation (3.5) can be used to determine the relative phases of the vertical velocities. The vertical velocities associated with the viscous motions at the edges of $V_{-}$and $z / L_{0} \rightarrow 0$ are determined from continuity so that on taking the horizontal divergence of (3.5), and taking care with the signs, the vertical $(V, S)$ velocities are found to be related by

$$
\rho_{-} w_{-}^{(V, S)}=\rho_{+} w_{+}^{(V, S)} \text {. }
$$

That is, the vertical 'pumping' velocities have the same sign. As discussed in $\S 6$, once all the components of the solution are added together the phasing is rather different: 
upwelling in the upper fluid is located above upwelling in the lower fluid. This phase relationship promotes scalar transport across the interface.

\subsection{Turbulence driven in the upper layer: regime (iii)}

If the turbulence is now driven in the upper layer, with the same conditions of small Froude number and large density jump, then there is a qualitative difference in the structure of turbulence across the interface - not merely a $180^{\circ}$ reflection - as shown schematically in figure $2(b)$.

The velocity fields are expressed as

$$
\begin{gathered}
\boldsymbol{u}_{+}=\boldsymbol{u}_{+}^{(H)}+\boldsymbol{u}_{+}^{(S)}+\boldsymbol{u}_{+}^{(V)}+\boldsymbol{u}_{+}^{(V, S)}, \quad z>0, \\
\boldsymbol{u}_{-}=\boldsymbol{u}_{-}^{(V)}+\boldsymbol{u}_{-}^{(V, S)}, \quad z<0 .
\end{gathered}
$$

The thicknesses of the viscous and source layers are the same, but the higher density and viscosity of the fluid in the lower layer reduce the velocity fluctuations at the interface, denoted $\boldsymbol{u}_{I}$, to a value much less than the driving turbulence. On referring to figure $2(b)$, we see that the velocity at the bottom of the upper source layer, $S_{+}$, is $\boldsymbol{u}_{B}$ and the velocity at the interface is $\boldsymbol{u}_{I}$. The change in velocity across the viscous layer $V_{+}$is $\boldsymbol{u}_{+}^{(V)}$. The motions in the lower source layer, $S_{-}$, are small, and therefore the change in velocity across the lower viscous layer, $V_{-}$, is $\boldsymbol{u}_{I}$. Scaling estimates for $\boldsymbol{u}_{I}$ and $\boldsymbol{u}_{+}^{(V)}$ can be found by matching the fluid velocity and stress at the interface, using the methods in $\S 3.3$, which yields

$$
\begin{gathered}
\boldsymbol{u}_{I} \sim \boldsymbol{u}_{B} /(1+R), \\
\boldsymbol{u}_{+}^{(V)} \sim-\boldsymbol{u}_{B} /\left(1+R^{-1}\right) .
\end{gathered}
$$

The solutions are similar to (3.4) for turbulence driven in the lower layer, but with a $180^{\circ}$ reflection and with $R \rightarrow R^{-1}$. Hence at the interface the velocity fluctuations are much reduced, and the coupling across the interface is weaker by a factor $R^{-1}$ when compared with the case in which turbulence is driven in the lower layer. As in $\S 3.4$, non-zero divergence in the lower viscous layer drives irrotational motion in a lower source layer, $S_{-}$, of magnitude

$$
\boldsymbol{u}_{-}^{(V, S)} \sim \boldsymbol{u}_{-}^{(V)}\left(\delta_{-} / L_{o}\right) \sim \boldsymbol{u}_{I}\left(\delta_{-} / L_{0}\right) \sim R^{-1} \boldsymbol{u}_{0}\left(\delta_{-} / L_{0}\right),
$$

again a factor $R^{-1}$ smaller than the coupling when the turbulence is driven in the lower layer.

Over longer periods of time, if the Reynolds number of the fluctuations in the lower viscous layer is large enough (i.e. $u_{-}^{(V)} \delta_{-}>10^{2}$ ) inflection points in this layer produce a layer of turbulent eddies which spreads downwards and whose length scale $L_{x}$ grows with depth,

$$
u_{-}^{\prime} \sim u_{I}\left(-z / \delta_{-}\right)^{-1}, \quad L_{x} \sim(-z), \quad \text { for } z<-\delta_{-}
$$

(Thompson \& Turner 1975) (see figure 2c). In practice this layer will be constrained by stratification or Coriolis effects.

\section{Modal solutions}

The velocity field is expressed as a Fourier series, assuming horizontal homogeneity:

$$
\boldsymbol{u}(\boldsymbol{x}, t)=\sum_{\boldsymbol{k}} \hat{\boldsymbol{u}}\left(k_{1}, k_{2}, z, t\right) \exp \left[\mathrm{i}\left(k_{1} x+k_{2} y\right)\right],
$$


(a)

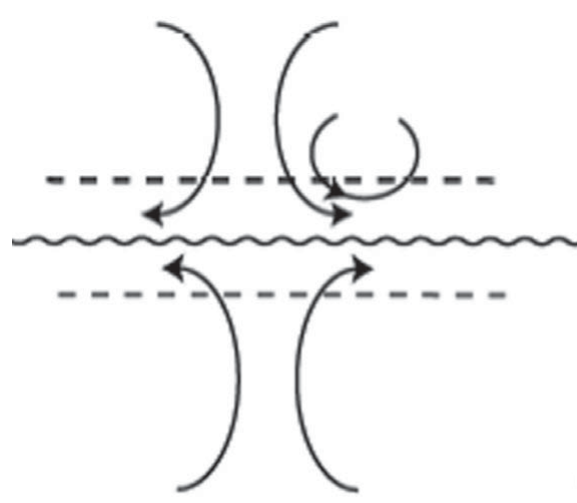

(b)
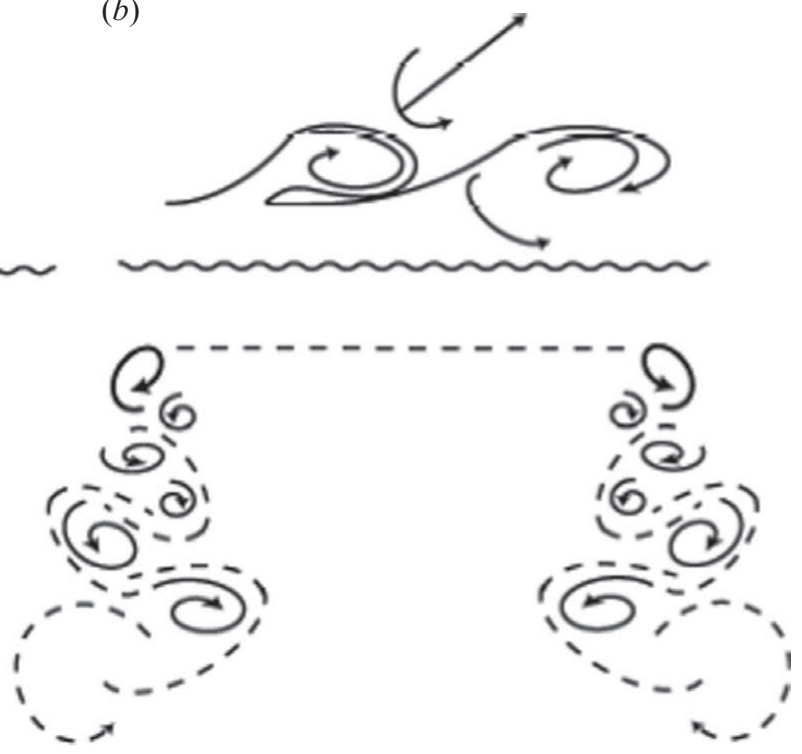

(c)

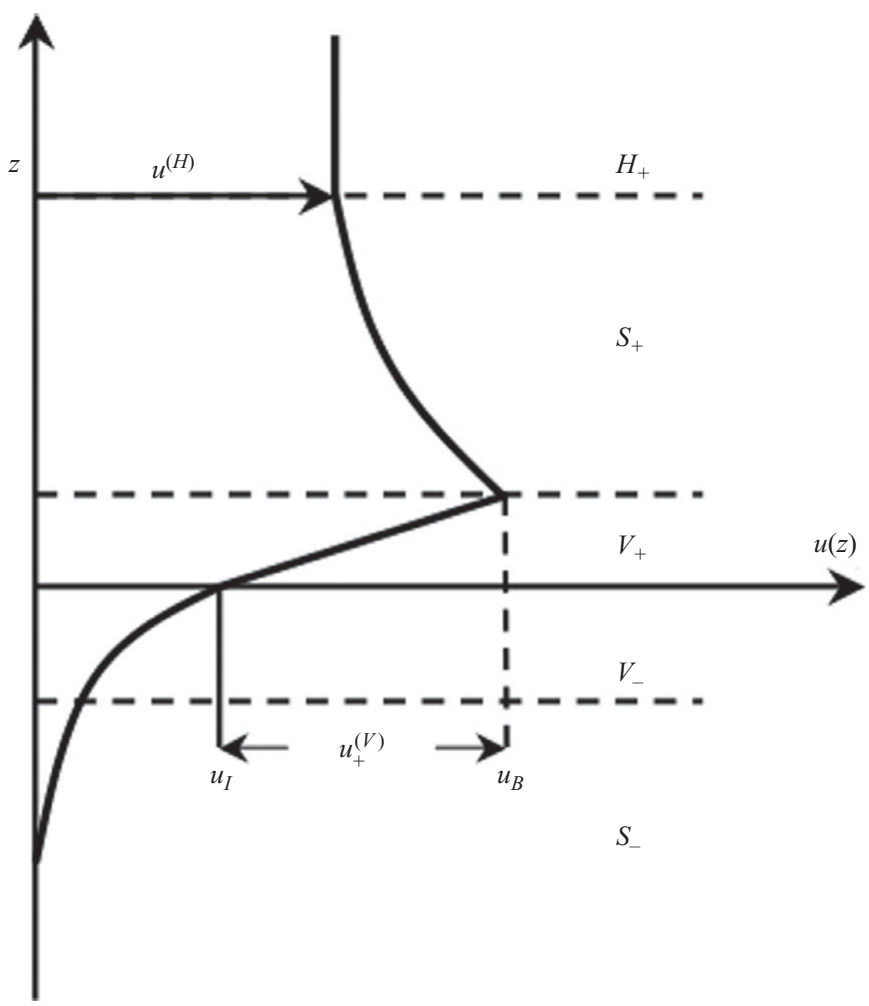

FiguRE 2. Schematic diagrams of $(a)$ the eddy structure distorted by linear processes, $(b)$ the eddy structure distorted by nonlinear processes (e.g. by shear in the upper fluid and nonlinear eddy-eddy interaction in the lower fluid) and $(c)$ the velocity profile for regime (iii) when turbulence is forced in the upper fluid. 
where $\hat{\boldsymbol{u}}$ are the Fourier components at each wavenumber $\boldsymbol{k}$. We initiate the analysis by calculating the response to a single Fourier mode of turbulence $\hat{\boldsymbol{u}}_{-}^{(H)}$ in the lower liquid layer to the sudden imposition of the interface at $z=0$ and $t=0$. Since we are using a linearized analysis, we can subsequently use superposition to combine a spectrum of such modes to represent a general turbulent velocity field (e.g. Townsend 1976). As before, it is assumed that $\rho_{-} / \rho_{+} \gg 1$ and $F_{I} \ll 1$ so that the interface remains flat.

\subsection{Turbulence driven by the lower layer: regime ( $i$ )}

In the homogeneous region $H_{-}$far below the interface $-z / L_{0} \gg 1$, the turbulence is unaffected by the interface so that $\boldsymbol{u}_{-}(\boldsymbol{x}, t) \rightarrow \boldsymbol{u}_{-}^{(H)}(\boldsymbol{x}, t)$, where the contributions from the regions $S_{-}$and $V_{-}$are zero. Following $\mathrm{HG}$, for times small compared with the Lagrangian time scale of the turbulence, $t \ll T_{0}=L_{0} / u_{0}$, the velocity fields in this region remains unchanged. Hence

$$
\boldsymbol{u}_{-}^{(H)}(\boldsymbol{x}, t)=\boldsymbol{u}_{-}^{(H)}(\boldsymbol{x}, 0) .
$$

In the $H_{-}$and $S_{-}$regions the vorticity is also unchanged so that

$$
\omega_{i-}(\boldsymbol{x}, t)=\omega_{i-}(\boldsymbol{x}, 0)=\omega_{i-}^{(H)}(\boldsymbol{x}, 0) .
$$

By considering a single plane-wave Fourier mode in the homogeneous region given by $\boldsymbol{u}_{-}^{(H)}(\boldsymbol{x}, t)=\hat{\boldsymbol{u}}_{-}^{(H)}(\boldsymbol{k}, 0) \exp \left[\mathrm{i}\left(k_{1} x+k_{2} y+k_{3} z\right)\right]$, we now calculate the flow resulting from this homogeneous mode in the other regions of the flow.

\subsubsection{Source layer $S_{-}$}

At times $t \geqslant 0^{+}$the velocity field in the source region, outside the interfacial viscous region, is given by

$$
\boldsymbol{u}_{-}(\boldsymbol{x}, t)=\boldsymbol{u}_{-}^{(H)}(\boldsymbol{x}, 0)+\boldsymbol{u}_{-}^{(S)}(\boldsymbol{x}, 0)+\boldsymbol{u}_{-}^{(V, S)}(\boldsymbol{x}, t) .
$$

As explained above, the velocity component $\boldsymbol{u}_{-}^{(V, S)}$ arising from the viscous region is of order $u_{I}\left(\delta_{-} / L_{0}\right)$ and can be neglected to first order for large Reynolds numbers. From $(4.2 b)$ it follows that the velocity field $\boldsymbol{u}_{-}^{(S)}$ is irrotational and can be expressed in terms of a velocity potential, namely $\boldsymbol{u}_{-}^{(S)}(\boldsymbol{x})=-\nabla \phi_{-}^{(S)}(\boldsymbol{x})$, so that

$$
\nabla^{2} \phi_{-}^{(S)}(x)=0 .
$$

Its boundary conditions are $\nabla \phi_{-}^{(S)} \rightarrow 0$ where $z / L_{0} \rightarrow-\infty$ and $\boldsymbol{u} \cdot \boldsymbol{n}=0$ (where $\boldsymbol{n}$ is the unit normal) at $z / L_{0}=0$ and $t \geqslant 0$ so that $-\partial \phi_{-}^{(S)} / \partial z=w_{-}^{(S)}=-w_{-}^{(H)}$ on $z / L_{0}=0$. For a single mode, namely

$$
\phi_{-}^{(S)}(\boldsymbol{x})=\hat{\phi}_{-}^{(S)}\left(k_{1}, k_{2}, z\right) \exp \left[\mathrm{i}\left(k_{1} x+k_{2} y\right)\right],
$$

the amplitude $\hat{\phi}_{-}^{(S)}$ satisfies $\left(\partial^{2} / \partial z^{2}-k_{12}^{2}\right) \hat{\phi}_{-}^{(S)}=0$, where $k_{12}^{2}=k_{1}^{2}+k_{2}^{2}$. The solution subject to the above boundary conditions is

$$
\hat{\phi}_{-}^{(S)}(\boldsymbol{x})=\hat{w}_{-}^{(H)} \frac{\exp \left(k_{12} z\right)}{k_{12}} .
$$

Hence,

$$
\boldsymbol{u}_{-}^{(S)}=-\hat{w}_{-}^{(H)}\left(\frac{\mathrm{i} k_{1}}{k_{12}}, \frac{\mathrm{i} k_{2}}{k_{12}}, 1\right) \exp \left(k_{12} z\right) \exp \left[\mathrm{i}\left(k_{1} x+k_{2} y\right)\right] .
$$

Notice how $\boldsymbol{u}_{-}^{(S)} \rightarrow 0$ over a distance of order $k_{12}^{-1}$ from the interface. For a spectrum of modes where the most energetic length scales are of order $L_{0}$, these solutions 
show how the effects of the interface decay on a scale $L_{0}$ from the interface. Near the boundary, as $z / L_{0} \rightarrow 0$, after the introduction of the interface for $0<t \ll T_{0}$ the inviscid blocking solution follows from the above expression as

$$
\begin{aligned}
& \boldsymbol{u}_{B}=\left(\boldsymbol{u}_{-}^{(H)}+\boldsymbol{u}_{-}^{(S)}\right)(z \rightarrow 0)=\left[\left(\hat{u}_{-}^{(H)}+\hat{w}_{-}^{(H)} \frac{\mathrm{i} k_{1}}{k_{12}}\right),\left(\hat{v}_{-}^{(H)}+\hat{w}_{-}^{(H)} \frac{\mathrm{i} k_{2}}{k_{12}}\right), 0\right] \\
& \times \exp \left[\mathrm{i}\left(k_{1} x+k_{2} y\right)\right] .
\end{aligned}
$$

The blocking velocity has only horizontal components and is a function of only horizontal position, i.e. $\boldsymbol{u}_{B}(x, y)=\left(u_{B}, v_{B}, 0\right)$. This is used in the next section as a boundary condition for calculating the flow in the interfacial viscous regions $V_{-}$ and $V_{+}$.

\subsubsection{Viscous interfacial layers $V_{-}$and $V_{+}$}

In the previous section we calculated the kinematic blocking effect of the interface on the homogeneous component of the flow $\boldsymbol{u}_{-}^{(H)}$. At time $t=0^{+}$the introduction of the interface gives rise to a discontinuity in the horizontal velocity field at $z=0$. We now calculate the viscous diffusion of vorticity and horizontal momentum in the interfacial region, using linearized boundary layer theory and the assumption of large $\operatorname{Re}$ (as in $\mathrm{HG}$ ). In the boundary layer approximations, pressure variations across the thin viscous regions are negligible so that the horizontal velocity components satisfy

$$
\frac{\partial \boldsymbol{u}_{ \pm}^{(V)}}{\partial t}=v_{ \pm} \frac{\partial^{2} \boldsymbol{u}_{ \pm}^{(V)}}{\partial z^{2}} .
$$

Similarity solutions for the interfacial region subject to continuity of the velocity and horizontal shear stress $\left(\tau_{x}, \tau_{y}\right)=\mu \partial / \partial z(u, v)$ at $z=0$ have been obtained with the initial and boundary conditions given by

$$
\boldsymbol{u}_{-}^{(V)}=\boldsymbol{u}_{B} \text { at } t=0^{+}, \quad z \leqslant 0
$$

and

$$
\boldsymbol{u}_{-}^{(V)} \rightarrow \boldsymbol{u}_{B} \text { as } z / \delta_{-} \rightarrow-\infty \text { and } \boldsymbol{u}_{+}^{(V)} \rightarrow 0 \text { as } z / \delta_{+} \rightarrow \infty,
$$

where $\delta_{-}=\left(2 v_{-} t\right)^{1 / 2}$ and $\delta_{+}=\left(2 v_{+} t\right)^{1 / 2}$ are the vertical length scales of the viscous regions on either side of the interface. Matching the interface velocity and horizontal stress at $z=0$ leads to

$$
\boldsymbol{u}_{+}(\boldsymbol{x}, t)=\boldsymbol{u}_{I}\left(1-\operatorname{erf}\left(z / \delta_{+}\right)\right), \quad \text { for } z>0,
$$

and

$$
\boldsymbol{u}_{-}(\boldsymbol{x}, t)=\left\{\boldsymbol{u}_{B}+\left(\boldsymbol{u}_{I}-\boldsymbol{u}_{B}\right)\left(1+\operatorname{erf}\left(z / \delta_{-}\right)\right)\right\}, \quad \text { for } z<0 .
$$

Here $\boldsymbol{u}_{I}(x, y)=\left(u_{I}, v_{I}, 0\right)$ is the fluctuating velocity at the interface, which, like $\boldsymbol{u}_{B}(x, y)=\left(u_{B}, v_{B}, 0\right)$, has only horizontal components, and therefore $\boldsymbol{u}_{-}$and $\boldsymbol{u}_{+}$also have only horizontal components in the viscous layers. Furthermore, $\boldsymbol{u}_{I}$ is given by

$$
\boldsymbol{u}_{I}=\frac{R}{1+R}\left(\boldsymbol{u}_{B}-\frac{\sqrt{\pi}}{2} \delta_{-} \frac{\partial \boldsymbol{u}_{B}}{\partial z}\right),
$$

where we define $\partial \boldsymbol{u}_{B} / \partial z=\partial / \partial z\left(u_{-}^{(H)}+u_{-}^{(S)}\right)$ as $z / L_{0} \rightarrow 0$. Equation $(4.7 c)$ is consistent with (3.4a): $\delta_{-} \partial \boldsymbol{u}_{B} / \partial z \sim \boldsymbol{u}_{B} \delta_{-} / L_{0} \sim \boldsymbol{u}_{B} R e^{-1 / 2}$ so that to leading order $\boldsymbol{u}_{I}=\boldsymbol{u}_{B} R /$ $(1+R)$.

Note that the thickness of both viscous layers grows as $t^{1 / 2}$ because the unsteady inertial forces are balanced by the viscous growth rate of the layers and $\boldsymbol{u}_{B}$ is 
effectively constant on a time scale $T_{0}$. We neglect the small contributions from the (time-dependent) $\boldsymbol{u}^{(V, S)}$ component in calculating the interfacial viscous region. Note that as $R \rightarrow \infty, \partial \boldsymbol{u}_{-} / \partial z \rightarrow 0$ at $z=0$, as expected for a free surface.

For the boundary layer approximations used for calculating the viscous diffusion of horizontal momentum across the interfacial region we ignore the (small) vertical velocity, which in $V_{-}$has two parts: the blocking velocity $w_{B-}$ which increases with $|z|$, determined by homogeneous and source contribution near the interface, and the viscous part that is constant and is driven by the horizontal divergence of the viscous effects in $V_{-}$. By contrast, in $V_{+}, w_{+}$has just one part, which arises from the viscous effects. From (3.7) the mass transport associated with the two viscous contributions is equal:

$$
\begin{aligned}
& \rho_{+} w_{+}^{(V)}\left(z / \delta_{+} \rightarrow \infty\right) \rightarrow-\left(\nabla \cdot \boldsymbol{u}_{I}\right) \frac{\rho_{+} \delta_{+}}{\pi^{1 / 2}}, \\
& \rho_{-} w_{-}^{(V)}\left(z / \delta_{-} \rightarrow \infty\right) \rightarrow-\left(\nabla \cdot \boldsymbol{u}_{I}\right) \frac{\rho_{+} \delta_{+}}{\pi^{1 / 2}} .
\end{aligned}
$$

Note that $\boldsymbol{u}_{I}$ has only horizontal components. From (4.5), (4.7) and (4.8a) the full profile for a single mode is

$$
\begin{array}{r}
\hat{w}_{+}\left(z / \delta_{+}>0\right)=-\nabla \cdot \hat{u}_{B} \cdot \frac{R}{1+R}\left\{z\left(1-\operatorname{erf}\left(z / \delta_{+}\right)\right)+\frac{\delta_{+}}{\sqrt{\pi}}\left(1-\exp \left[-\left(z / \delta_{+}\right)^{2}\right]\right)\right\} \\
\times \exp \left(-k_{12} z\right), \quad(4.8 c)
\end{array}
$$

where $\nabla \cdot \hat{u}_{B}=\mathrm{i}\left\{k_{1}\left(\hat{u}^{(H)}+\hat{w}^{(H)} \mathrm{i} k_{1} / k_{12}\right)+k_{2}\left(\hat{v}^{(H)}+\mathrm{i} k_{2} \hat{w}^{(H)} / k_{12}\right)\right\}$.

\subsubsection{Source region $S_{+}$}

As shown in the previous section, a horizontal divergence in the interfacial velocity field gives rise to a vertical velocity field at the outer edge of the viscous regions. The viscous layer therefore acts like a source distribution which drives an irrotational velocity field in the region $S_{+}$above the interface. The velocity in this region, due to the turbulence below the interface, can therefore be written as $\boldsymbol{u}_{+}=\boldsymbol{u}_{+}^{(V, S)}$, where $\boldsymbol{u}_{+}^{(V, S)}=-\nabla \phi_{+}^{(V, S)}$ in which

$$
\nabla^{2} \phi_{+}^{(V, S)}=0
$$

The boundary conditions are

$$
\boldsymbol{u}_{+}^{(V, S)} \rightarrow 0 \quad \text { as } z / L_{0} \rightarrow \infty \quad \text { and } \quad w_{+}^{(V)}\left(z / \delta_{+} \rightarrow \infty\right)=-\left(\nabla_{H} \cdot \boldsymbol{u}_{I}\right) \frac{\delta_{+}}{\pi^{1 / 2}}
$$

The solution in $S_{+}$is (for a single mode)

$$
\boldsymbol{u}_{+}^{(V, S)}(\boldsymbol{x}, t)=-\left(\nabla \cdot \boldsymbol{u}_{I}\right) \frac{\delta_{+}}{\pi^{1 / 2}}\left(\frac{\mathrm{i} k_{1}}{k_{12}}, \frac{\mathrm{i} k_{2}}{k_{12}}, 1\right) \exp \left(-k_{12} z\right) \exp \left[\mathrm{i}\left(k_{1} x+k_{2} y\right)\right] .
$$

Note that since $\left|\boldsymbol{u}_{I}\right| \sim u_{0}$ the magnitude of $u_{+}$scales as $\left(\delta_{+} / L_{0}\right) u_{0}$ and decays away from the interface on a scale of order $L_{0}$ (the length scale of the homogeneous turbulence in $H_{-}$). 


\subsection{Turbulence driven by the upper layer: regime (iii)}

The analysis for this case (described in $\S 3.5$ ) when $R e \gg 1$ follows that in $\S 4.1$. Here $L_{0}$ defines the scale of the upper layer fluctuations. In the upper viscous layer, if the blocking component is defined as

$$
\boldsymbol{u}_{B}=\boldsymbol{u}_{+}^{(H)}(z \rightarrow 0)+\boldsymbol{u}_{+}^{(S)}(z \rightarrow 0)
$$

the horizontal component is given by matching the stress and the horizontal components of velocity at $z=0$. Then the horizontal velocity field in $V_{+}$is

$$
\boldsymbol{u}_{+}(\boldsymbol{x}, t)=\left\{\boldsymbol{u}_{B}+\left(\boldsymbol{u}_{I}-\boldsymbol{u}_{B}\right)\left(1-\operatorname{erf}\left(z / \delta_{+}\right)\right)\right\},
$$

where the velocity at the interface is given by

$$
\boldsymbol{u}_{I}=\frac{1}{1+R} \boldsymbol{u}_{B}
$$

Below the interface

$$
\boldsymbol{u}_{-}(\boldsymbol{x}, t)=\boldsymbol{u}_{I}\left(1+\operatorname{erf}\left(z / \delta_{-}\right)\right) \text {. }
$$

For $z<-\delta_{-}$, there is a weak irrotational flow driven by the divergence in the lower viscous layer (i.e. in the linear regime when $t<T_{0}$ ):

$$
\boldsymbol{u}_{-}=\boldsymbol{u}_{-}^{(V, S)} \sim \hat{\boldsymbol{u}}_{-}^{(V, S)} \mathrm{e}^{-k_{12} z},
$$

where (as in (4.10)) $\left|\hat{\boldsymbol{u}}^{(V, S)}\right| \sim\left(\delta_{-} / L_{o}\right)\left|\boldsymbol{u}^{(V)}\right| \sim\left(\delta_{-} / L_{o}\right) u_{I}$.

\section{Statistics of the inhomogeneous turbulence}

\subsection{Spectrum of the forcing turbulence}

Once either assumptions or measurements are made to specify the forcing turbulence the modal solutions derived above can be used to calculate statistics of the inhomogeneous velocity fields near the interface. For simplicity assume that the turbulence far from the interface, $\boldsymbol{u}^{(H)}$, is isotropic with a spectrum tensor of the form $\Phi_{i j}(\boldsymbol{k})=\left(\delta_{i j}-\left(k_{i} k_{j} / k^{2}\right)\right)\left(E(k) / 4 \pi k^{2}\right)$, where $k=|\boldsymbol{k}|=k_{i} k_{i}$ and $E(k)$ is the spectral energy density function. This assumption can be generalized to an anisotropic turbulence, following Nagata et al. (2006). HG used two forms for the spectral energy density, namely

$$
E(k)=\frac{u_{0}^{2} g_{1} k^{4} L_{0}^{5}}{\left(1+g_{2} k^{2} L_{0}^{2}\right)^{17 / 6}},
$$

where $g_{1}$ and $g_{2}$ are coefficients, which represents very high-Reynolds-number turbulence, and

$$
E(k)=\frac{8}{\pi} \frac{u_{0}^{2} k^{4} L_{0}^{5}}{\left(1+k^{2} L_{0}^{2}\right)^{3}}
$$

which represents moderate-Reynolds-number turbulence. These forms are suitable for calculating the statistics of the blocking solutions in $S_{-}$for regime (i) or $S_{+}$for regime (iii). However, for the viscous interfacial layer, the model developed here assumes that the scales of the impinging eddies are larger than the thickness of the viscous layer (which is of the order of the Taylor micro-scale). The smaller, inertial-range eddies do not penetrate the vortical viscous layer (e.g. Zaki \& Saha 2009), because of the shear sheltering mechanism (Hunt \& Durbin 1999). It is therefore consistent to use a spectral form with a rapid decay for high wavenumbers in order to concentrate on the 
interaction of the larger, energy-containing scales. The exponential form of spectrum approximates to laboratory experiments and moderate-Reynolds-number numerical simulations (Townsend 1976; Nagata et al. 2006), i.e.

$$
E(k)=\frac{1}{(2 \pi)^{1 / 2}} u_{0}^{2} k^{4} L_{0}^{5} \exp \left(-\frac{1}{2} k^{2} L_{0}^{2}\right) .
$$

We examine regimes (i) and (iii) where $\boldsymbol{u}_{+}^{(H)}=0$ and $\boldsymbol{u}_{-}^{H}=0$, respectively. Thus initially the turbulence exists only below or above the interface. The derivation of statistics for cases including turbulence on both sides of the interface can be obtained by straightforward superposition, provided $\boldsymbol{u}_{-}^{(H)}$ and $\boldsymbol{u}_{+}^{(H)}$ are uncorrelated.

\subsection{Mean square velocity fluctuations in the source and viscous layers}

Expressions for the mean square velocity fluctuations in the various layers of the flow follow from the modal solutions derived in $\S 4$. The results in the source layers, $S_{-}$ and $S_{+}$, are the same as calculated in $\mathrm{HG}$, if we ignore the small contributions from $\boldsymbol{u}_{-}^{(V, S)}$, which are $O\left(R e^{-1 / 2}\right)$ (see also Calmet \& Magnaudet 2003 for measurements). In the limit $z / L_{0} \rightarrow 0$, but outside the viscous region $V_{-}, V_{+}$, the results for the source layer yield

$\overline{\boldsymbol{u}_{B}^{2}}=\overline{\left(u_{-}^{(H)}(0)+u_{-}^{(S)}(0)\right)^{2}}+\overline{\left(v_{-}^{(H)}(0)+v_{-}^{(S)}(0)\right)^{2}}=3 u_{0}^{2} \quad$ and $\quad \overline{\left(w^{(H)}(0)+w^{(S)}(0)\right)^{2}}=0$

for any type of homogeneous forcing turbulence $\boldsymbol{u}^{(H)}$, independent of the form of $E(k)$, including anisotropic turbulence and homogeneous shear flows near a plane boundary (Lee \& Hunt 1988; Mann 1994; Nagata et al. 2006). As (5.2) demonstrates there is a transfer of vertical kinetic energy into the horizontal components as the wall is approached. The full profiles of the velocity variances in the source regions do depend on the form of $E(k)$. The exponential cutoff spectrum (5.1c), with reduced energy at smaller scales, predicts much stronger damping of the vertical velocities near the wall when compared with the other spectral forms $(5.1 a, b)$. Turbulence measurements below a free surface were compared with theory by Hunt (1984) and Brutsaert \& Jirka (1984).

Consider now the solutions in the viscous layer, first in regime (i), when the forcing turbulence is in the lower layer. From $(4.7 a b)$, when the Reynolds number is sufficiently large so that $\delta_{-} \ll L_{0}$, the profile of the velocity variance is

$$
\begin{gathered}
\overline{\boldsymbol{u}_{+}^{2}}=\overline{\boldsymbol{u}_{I}^{2}}\left(1-\operatorname{erf}\left(z / \delta_{+}\right)\right)^{2}, \quad z>0, \\
\overline{\boldsymbol{u}_{-}^{2}}=\overline{\left(\boldsymbol{u}_{-}^{(H)}(z)+\boldsymbol{u}_{-}^{(S)}(z)\right)^{2}}+\overline{\boldsymbol{u}_{B-}^{2}} \cdot P_{-}(z), \quad z<0,
\end{gathered}
$$

where the projection operator is given by

$$
P_{-}(z)=\frac{1}{(1+R)^{2}}\left(1+\operatorname{erf}\left(z / \delta_{-}\right)\right)^{2}-2 \frac{1}{1+R}\left(1+\operatorname{erf}\left(z / \delta_{-}\right)\right)
$$

and

$$
\overline{\boldsymbol{u}_{I}^{2}}=\overline{\boldsymbol{u}_{B-}^{2}} R^{2} /(1+R)^{2} .
$$

Figure 3 shows profiles of the r.m.s. of the horizontal components of velocity variance, $u^{\prime} / u_{0}=\left(\overline{u^{2}}\right)^{1 / 2} / u_{0}$, when the forcing turbulence is isotropic. 


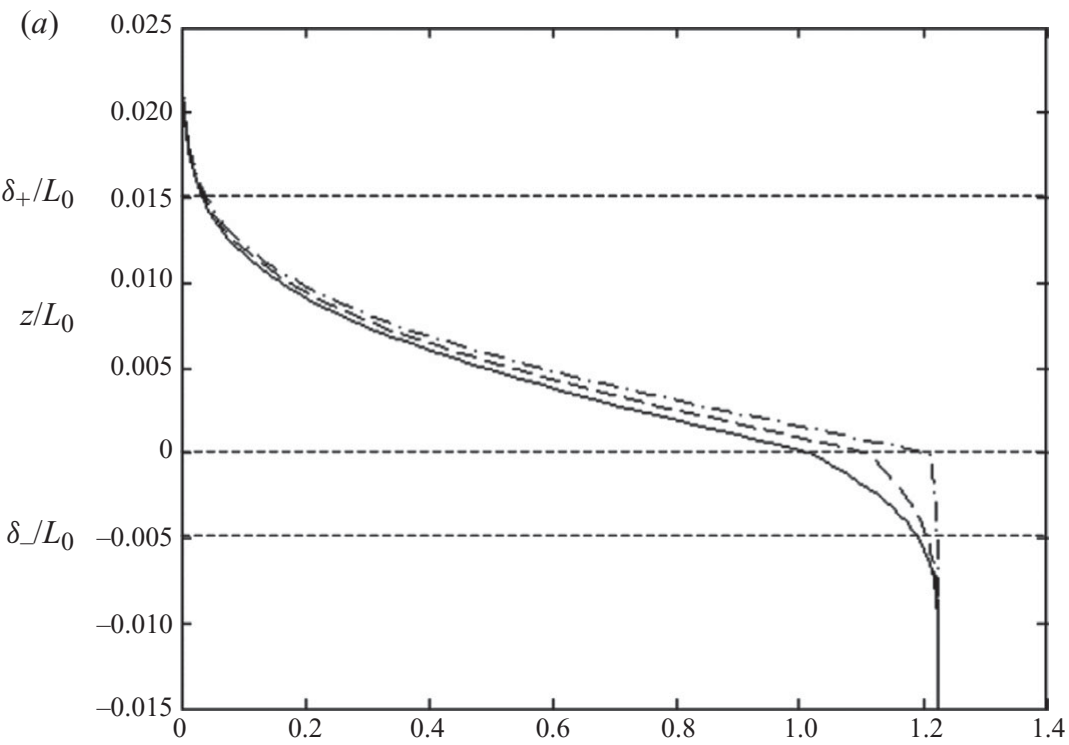

(b)

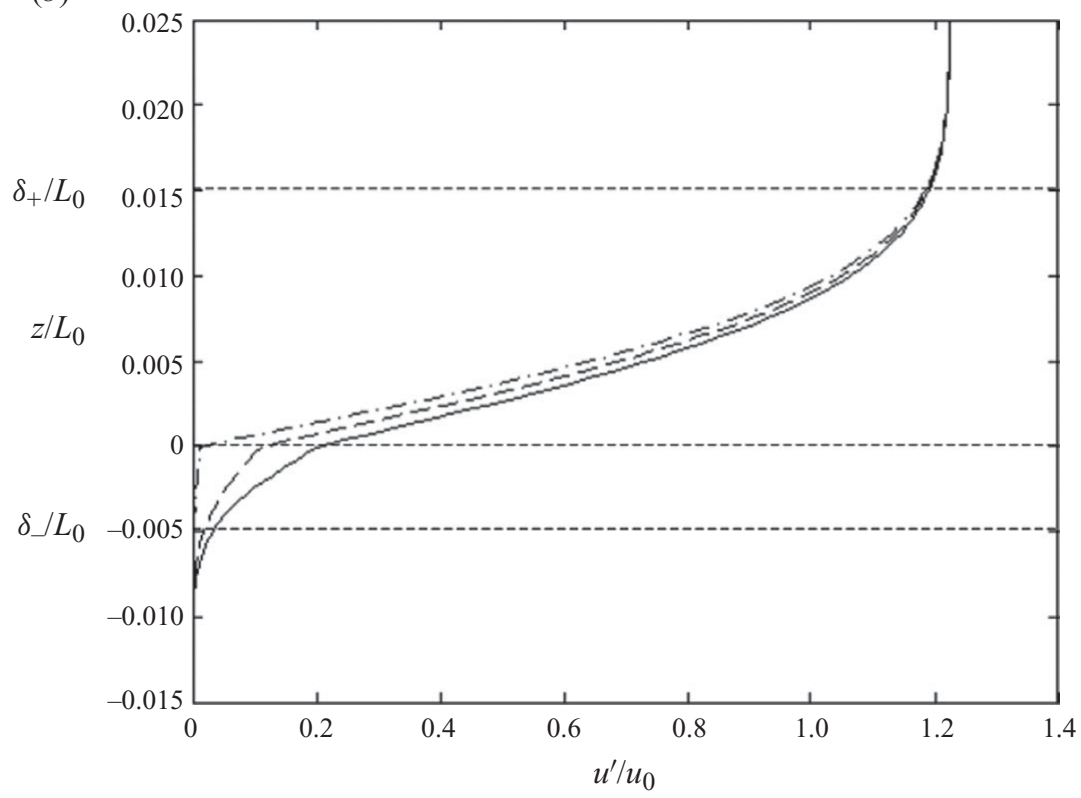

FIGURE 3. Vertical profiles of the horizontal velocity fluctuations across the viscous layers, when isotropic turbulence is forced in $(a)$ the lower fluid and $(b)$ the upper fluid: solid line, $R=5$; dashed line, $R=10$; dot-dashed line, $R=100$.

Figure 3(a) shows the case in which isotropic turbulence is forced in the lower layer, and figure $3(b)$ shows the one in which isotropic turbulent is forced in the upper layer. In each case, results are shown for $\delta_{+} / L_{0}=0.01$ and $\delta_{-} / L_{0}=0.005$ (so that, for example, if $L_{0}=1 \mathrm{~m}$ and $u_{0}=1 \mathrm{~m} \mathrm{~s}^{-1}$, then $v_{+}=5 \times 10^{-5} \mathrm{~m}^{2} \mathrm{~s}^{-1}$ and $\nu_{-}=1.25 \times 10^{-5} \mathrm{~m}^{2} \mathrm{~s}^{-1}$ ) and for $R=5,10$ and 100 (so that, for example, if $\rho_{+}=1 \mathrm{~kg} \mathrm{~m}^{-3}$, then $\rho_{-}=10,20$ or $200 \mathrm{~kg} \mathrm{~m}^{-3}$ ). 
The profiles in figure $3(a)$ show that when $R$ is large, the velocity profile within the lower layer is affected little by the upper fluid even in the viscous layer. If the forcing turbulence is isotropic so that the $k$ th component of the forcing turbulence has variance $\overline{\left(\boldsymbol{u}_{k}^{(H)}\right)^{2}}=u_{0}^{2}$, then the blocking velocity is the same in each horizontal direction and, from (5.2), is given by $\overline{u_{B}^{2}}=(3 / 2) u_{0}^{2}$. The variance of the interfacial velocity is then also the same in each horizontal direction and is given by

$$
\overline{u_{I}^{2}}=\frac{3}{2} \frac{R^{2}}{(1+R)^{2}} u_{0}^{2} .
$$

Hence, when $R$ is large, $u^{\prime} / u_{0} \approx(3 / 2)^{1 / 2} \approx 1.22$ right up to the interface. Deeper into the lower layer the blocking effect of the interface decreases, and the velocity fluctuations reduce to $u^{\prime} / u_{0}=1$ over depths greater than the integral length scale. For the smaller values of $R$ the velocity fluctuations across the viscous layer reduce to the value of the interfacial fluctuations. Hence, since the fluctuations also decay with depth, the maximum velocity fluctuations are in the lower fluid interior, just below the viscous layer at $z \approx-2 \delta_{-}$. Figure $3(a)$ also shows that velocity fluctuations, of order $u_{I}$, are also driven in the upper fluid and decay over the depth of the upper viscous layer. Above that are only the very small $(V, S)$ solutions.

Consider now regime (iii), when the turbulence is forced in the upper fluid. From $(4.12 a, b, c)$ for $\delta_{+} \ll L_{0}$,

$$
\begin{gathered}
\overline{\boldsymbol{u}_{+}^{2}}=\overline{\left(\boldsymbol{u}_{+}^{(H)}(z)+\boldsymbol{u}_{+}^{(S)}(z)\right)^{2}}+\overline{\boldsymbol{u}_{B+}^{2}} \cdot P_{+}(z), \quad z>0, \\
\overline{\boldsymbol{u}_{-}^{2}}=\frac{\overline{\boldsymbol{u}_{B+}^{2}}}{(1+R)^{2}}\left(1+\operatorname{erf}\left(z / \delta_{-}\right)\right)^{2}, \quad z<0,
\end{gathered}
$$

where the projection operator is given by

$$
P_{+}(z)=\frac{R^{2}}{(1+R)^{2}}\left(1-\operatorname{erf}\left(z / \delta_{-}\right)\right)^{2}-2 \frac{R}{1+R}\left(1-\operatorname{erf}\left(z / \delta_{-}\right)\right)
$$

and

$$
\overline{\boldsymbol{u}_{I}^{2}}=\overline{\boldsymbol{u}_{B+}^{2}} /(1+\mathrm{R})^{2}
$$

Following the reasoning leading to (5.3e), when the forcing turbulence is isotropic, the variance of the interfacial velocity is the same in each direction and is given by

$$
\overline{u_{I}^{2}}=\frac{3}{2} \frac{1}{(1+R)^{2}} u_{0}^{2}
$$

Figure $3(b)$ shows vertical profiles of the velocity fluctuations when the turbulence is forced in the upper fluid. As in the case of turbulence forced in the lower fluid, turbulent fluctuations increase towards the interface through the blocking mechanism and are then reduced across the viscous layer, leading to maximum velocity fluctuations just above the upper viscous layer. There is a qualitative difference, however, from the case of turbulence forced in the lower fluid, namely that the fluctuations at the interface are small for large values of $R$. Consequently, fluctuations in the lower fluid are also small.

\subsection{Cross-correlations near the interface}

The RDT solutions can be used to investigate cross-correlations between the velocity fields on the same or different sides of the interface. Following Hunt (1984), we define 
(a)

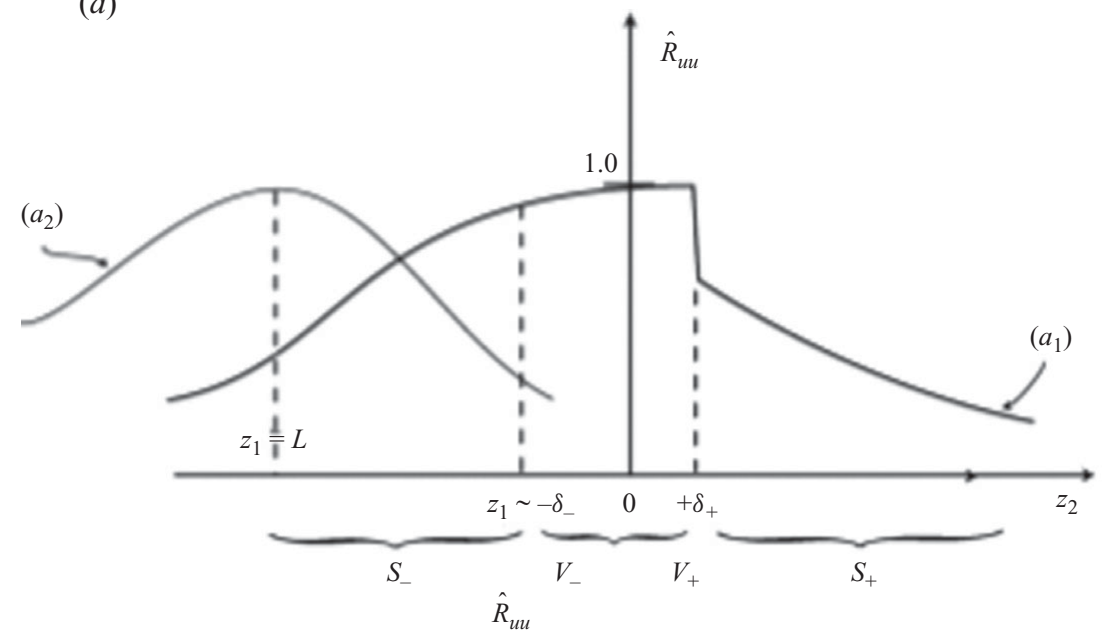

(b)

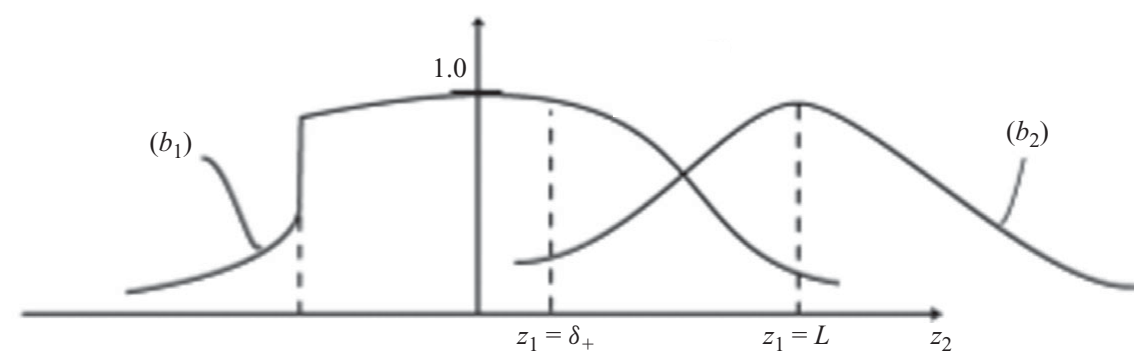

FIGURE 4. Sketches of the cross-correlation of the horizontal velocity, normalized by their value at $z_{1}$, when isotropic turbulence is forced in $(a)$ the lower fluid and $(b)$ the upper fluid: curve $\left(a_{1}\right), z_{1}=-\delta_{-} ;$curve $\left(a_{2}\right), z_{1}=-L_{0}$; curve $\left(b_{1}\right), z_{1}=\delta_{+} ;$curve $\left(b_{2}\right), z_{1}=L_{0}$.

the cross-correlation between the horizontal velocity at $z_{1}$ and $z_{2}$ to be normalized by the velocity at one point, namely

$$
\hat{R}_{u u}=\frac{\overline{u\left(z_{1}\right) u\left(z_{2}\right)}}{\overline{u^{2}}\left(z_{1}\right)} .
$$

The magnitude of this correlation indicates the strength of coupling between the turbulence on either side of the interface. If $z_{1}=\delta_{-}$, then the cross-correlation can be used to investigate coupling of the flows near the interface, such as the streak motions in the coupled viscous sublayers, described in Lombardi et al. (1996) and Lee, Moin \& Kim (1997). The solutions developed in $\S 4$ demonstrate that there is a strong correlation between fluctuations across the interface within the viscous layers when turbulence is forced in the lower layer, and if $z_{1}=\delta_{-}$, then

$$
\begin{gathered}
\hat{R}_{u u}\left(z_{1}=\delta_{-}, z\right)=\frac{R}{1+R}\left(1-\operatorname{erf}\left(z / \delta_{+}\right)\right), \quad z>0, \\
\hat{R}_{u u}\left(z_{1}=\delta_{-}, z\right)=1-\frac{1}{1+R}\left\{1+\operatorname{erf}\left(z / \delta_{-}\right)\right\}, \quad-\delta_{-}<z<0 .
\end{gathered}
$$

Profiles are sketched in figure $4(a)$. Across the viscous layers there is a strong correlation, which drops towards the outer edge of the viscous boundary layer in 
the upper fluid. Above this layer there remains a small correlation with the $(V, S)$ components of the motion. The correlation with horizontal fluctuations below the viscous layer decays through the blocking layer over the integral length scale of the forcing turbulence.

When the turbulence is forced in the upper layer and $z_{1}=\delta_{+}$the cross-correlation across the viscous layers is much weaker:

$$
\begin{gathered}
\hat{R}_{u u}\left(z_{1}=\delta_{-}, z\right)=1-\frac{R}{1+R}\left(1-\operatorname{erf}\left(z / \delta_{+}\right)\right), \quad \delta_{+}>z>0, \\
\hat{R}_{u u}\left(z_{1}=\delta_{-}, z\right)=\frac{1}{1+R}\left\{1+\operatorname{erf}\left(z / \delta_{-}\right)\right\}, \quad z<0 .
\end{gathered}
$$

Profiles are sketched in figure $4(b)$. The correlation remains large across the upper viscous layer, but because the interfacial fluctuations are small when $R$ is large (5.4e), it drops markedly across the interface. We return to this finding in connection with previous computations in $\S 6$.

Consider now the correlations in the vertical velocity. Since the vertical velocity fluctuations are small across the interface, it is necessary to use a different measure of the cross-correlation. Here we use the two-point velocity correlation normalized on the r.m.s. velocity at each point, namely

$$
R_{w w}\left(z_{1}, z_{2}\right)=\frac{\overline{w\left(z_{1}\right) w\left(z_{2}\right)}}{\left(\overline{w^{2}\left(z_{1}\right)} \overline{w^{2}\left(z_{2}\right)}\right)^{1 / 2}} .
$$

Consider the case of turbulence forced in the lower fluid. Following the analysis through demonstrates that a region of upwelling in the lower layer leads to divergence at the interface through the blocking mechanism. Viscous coupling across the interface then leads to divergence in viscous layer in the upper fluid and therefore to a region of downwelling in the source layer above. This set-up is shown schematically in figure $1(a)$. In this case $R_{w w} \rightarrow-1$, and the vertical velocity fluctuations are in perfect anti-correlation within the interfacial viscous regions and decay to zero exponentially over length scale $L_{0}$ in the regions on either side of the interface. The same reasoning follows through to the case in which turbulence is forced in the upper fluid.

\section{Estimates of nonlinear effects and comparison with simulation}

If the Reynolds number is high enough, the shearing motions in the viscous layers become unstable, and a new structure of the interface turbulence emerges. The results of direct numerical simulation studies for the different regimes provide useful insight into the nonlinear mechanisms.

In regime (i), when the turbulence is generated in the liquid layer, the Reynolds number of the fluctuations in the viscous layer below the surface is

$$
R e_{\delta_{-}}^{(i)}=\frac{\Delta u \delta_{-}}{v_{-}}
$$

where the velocity difference across the layer, $\Delta u=u_{I} / R$, is the appropriate velocity scale to diagnose instability of the layer. Since $\delta_{-} \sim \sqrt{T_{0} \nu_{-}} \sim L_{0} R e_{-}^{-1 / 2}$, it follows that

$$
R e_{\delta_{-}}^{(i)} \sim R e_{-}^{1 / 2} / R
$$


For the viscous layer in the lower fluid

$$
R e_{\delta_{+}}^{(i)}=\frac{u_{I} \delta_{+}}{v_{+}} \sim R e_{-}^{1 / 2}\left(\frac{\nu_{-}}{v_{+}}\right)^{1 / 2}
$$

For an air-water interface, when $R$ is approximately 300 , and for typical geophysical parameter values where $R e_{-} \sim 10^{4}-10^{5}$, the Reynolds number of the water-side boundary layer is much smaller than in the bulk of the flow, and additional turbulence is typically not generated there. The Reynolds number of the air-side boundary layer is typically large enough to generate turbulence, and thence turbulence in the upper layer.

In regime (iii), when turbulence is generated in the upper layer, the liquid acts almost like a rigid surface because $u_{I} \sim u_{0+} / R$. The Reynolds number of the upper viscous layer is then

$$
R e_{\delta_{+}}^{(i i i)}=\frac{u_{0+} \delta_{+}}{v_{+}} \sim R e_{+}^{1 / 2}
$$

Therefore, for typical engineering or geophysical flows, the boundary layer in the upper fluid generates its own turbulent eddies.

The liquid layer fluctuations are small compared with those in the gas layer for many engineering flows. For example, for air-water flow where $R \approx 300$ and $R e_{+} \sim 10^{3}$, $u_{0-} / u_{*+} \leqslant 1 / 10$, even for large values of $R e_{+} \sim\left(10^{6}-10^{7}\right)$.

Do these liquid layer fluctuations become unstable and develop their own turbulence? The Reynolds number of fluctuations in the viscous layer in the liquid for high-Reynolds-number gas flows can be estimated:

$$
R e_{\delta_{-}} \leqslant u_{-} \delta_{-} / \nu_{-} \sim \frac{u_{*+} \sqrt{L_{+} \nu_{-} / u_{*+}}}{\nu_{-}} \sim R e_{+}^{1 / 2}\left(\frac{\nu_{+}}{v_{-}}\right)^{1 / 2}, \quad \text { if } u_{-} \sim u_{+} .
$$

Hence, for the highly energetic and/or large-scale turbulence in the gas where $R e_{+} \sim 10^{7}$, the Reynolds number $R e_{\delta_{-}}$of the fluctuations in the liquid below the surface is of order $10^{3}$. Since the profile of the liquid layer velocity fluctuations $u_{-}$ has inflection points (see figure 1) a significant level of turbulence can be generated.

In some situations a mixed regime develops, when turbulent flows are generated in both the liquid and gaseous layers at a sufficiently high Reynolds number such that both flows are fully turbulent. They are coupled at the interface. A statistically steady state can develop where the velocity fluctuations and shear stresses are continuous so that

$$
\rho_{+} u_{*+}^{2} \sim \rho_{-} u_{*-}^{2} .
$$

Since $\rho_{-} \gg \rho_{+}$, this implies that $u_{*_{-}} \ll u_{*+}$. As our analysis of regimes (i) and (iii) ((4.7) and (4.12) respectively) show, the velocity fluctuations at the interface are of the order of the velocity fluctuations in the liquid layer $u_{-}$. (For typical high-Reynoldsnumber eddies in a shear-free flow near a resistive surface the fluctuating shear stress velocity $u_{*+}$ is proportional to but smaller than $u_{0+}$; Zilitinkevich et al. 2006.) But the relation between $u_{*}$ and $u_{0}$ in the upper and lower layers depends on the type of turbulent flow and the Reynolds number and also on the boundary conditions on the flows above and below the interface (especially for shear-free turbulence in the liquid layer).

The results are now compared with direct numerical simulations from Lombardi et al. (1996). They considered turbulent pressure-driven flows in a domain half filled with gas and half filled with liquid with the interface constrained to be flat 
but across which the horizontal velocity and shear stress were continuous. They performed simulations and investigated the turbulence structure in each case for various values of the ratio of the fluid densities, namely $\rho_{-} / \rho_{+}=1,100$ and 900 (the last of which corresponds approximately to an air-water interface, where $v_{-} / v_{+}=1 / 10$ and $\mu_{+} / \mu_{-} \sim 10^{-2}$ ). When there are turbulent shear flows in the liquid and gas layers (as in the numerical simulations of Lombardi et al. 1996), the shear stresses are proportional to the velocity variances, i.e. $u_{*+} \sim u_{+}$and $u_{*_{-}} \sim u_{-}$(Townsend 1976). Therefore, from $(6.4 a)$

$$
u_{-} \sim\left(\frac{\rho_{+}}{\rho_{-}}\right)^{1 / 2} u_{+}
$$

Since these two fields of turbulence are generated independently above and below the interface both fields are blocked by the interface, both of them generate fluctuations on the opposite side of the interface so that near the interface the flows are correlated. Note that the blocking effects at an interface are similar whether there is a mean shear or not (Lee \& Hunt 1988). The interaction between these blocked fields across the interface can be studied using our shear-free model, with the assumption that $u_{0-} / u_{0+}$, the velocity fluctuations above and below the interface, are proportional to $u_{*_{-}} / u_{*+}$. Therefore from $(6.4 b)$

$$
u_{0-} / u_{0+} \approx\left(\frac{\rho_{+}}{\rho_{-}}\right)^{1 / 2} \text {. }
$$

Since from $(3.4 a)$ the interface velocity fluctuation is equal to the liquid layer fluctuations (when $R \gg 1$ ), i.e. $u_{I} \approx u_{0-}$, it follows that the model predicts

$$
u_{I} / u_{0+} \approx\left(\frac{\rho_{+}}{\rho_{-}}\right)^{1 / 2}=0.0335 \text {, }
$$

which agrees with the results of the simulations of Lombardi et al. (1996), which yield a value of 0.0334 .

Note that to compare the simulations with our model results we consider only the spanwise velocity component $v$ because it is less affected by shear, and the mean square profiles do not vary strongly near any resistive surface; i.e. we assume $u_{0+}=\sqrt{\overline{\overline{u_{0}^{2}}}}$ and $u_{I}=\sqrt{\overline{u^{2}}}$, with the latter at $z=0$. Our model shows in this case that the turbulence on the air side contributes only about $3 \%$ to $u_{I}$ with the remaining $97 \%$ coming from the turbulence in the water below the interface. For this high density ratio, the turbulence on the denser side has a structure similar to that at a free-slip boundary. For $\rho_{-} / \rho_{+} \approx 100$, the simulations gave $v_{I} / u_{*+}=0.1$, when nondimensionalized on the friction velocity in the upper fluid. Again the model predictions are in agreement at 0.103 , with about $9 \%$ of the horizontal kinetic energy at $z=0$ coming from the upper less dense fluid and $91 \%$ from the lower fluid. These results indicate that the changes to the turbulence structure on the liquid side of the interface are not due to direct coupling from the gas turbulence but are a consequence of it changing, as it induces larger fluctuations above the interface (see also Csanady 1997, who focuses on the critical role of surface waves in sheared flow). Even though only a very small proportion of the velocity fluctuations in the liquid layer is induced by the turbulence in the upper layer, they have a distinct 'streaky' structure and extend over a significant distance of order $L_{0}$ into the liquid layer. Because of this coupling, as noted in the Introduction, a similar structure of elongated 'streaks' is observed in the liquid layer. But if the wind flow in the gaseous layer is large enough (regime (ii)), 
then waves are formed on the interface; these lead to a further change in the eddy structure (Thorpe 2004).

\section{Conclusions}

In this study we have investigated coupling across horizontal planar fluid interfaces (particularly gas-liquid interfaces) by extending previous work on the distortion of turbulence structure near plane boundaries by the blocking mechanism in the absence of mean shear. The analysis also provides a valid physical description of the kinematics and dynamics of large-scale energetic eddies near an interface and how they vary as the relative densities and viscosities and turbulence intensities change. Previous studies of this distorted eddy structure have emphasized how they control mass and heat transfer across these interfaces, starting with the surface renewal mechanism of Danckwert (1951). In the case of air-water interfaces, with shear-free turbulence driven on the water side of the interface (regime (i)), this leads to interfacial velocity fluctuations of the same order as $u_{0-}$, with some amplification by the blocking effect (Calmet \& Magnaudet 2003). The eddy structure in the liquid near the surface changes over a long period to the well-known form of vertical vortices (Tsai 1998). In this regime (regime (ii)), the velocity fluctuations generated above the interface are of the same order as those in the lower layer. Although they have a lower Reynolds number, they are generally turbulent.

When turbulence is driven in the low-density (or air) side above the interface (regime (iii)), our analysis shows that liquid-side fluctuations near the surface (for large $\left.\rho_{+} / \rho_{-}\right)$are in phase with the gas-side turbulence with a close correlation of the growth rates of interior viscous layers above and below the interface. The liquid-side fluctuations can become fully turbulent (at high Reynolds numbers $>10^{6}$ on the air side); but for moderate Reynolds numbers, the fluctuations on the liquid side become turbulent only if there is some additional forcing (e.g. mean shear, buoyant convection or surface waves). But even with separate forcing mechanisms above and below the surface, the vertical motion induced by the viscous layers at the interface ensures that the horizontal flow structures above and below the surface remain partly coupled. This explains the similarity of the streak structure on the gas and liquid sides of the interface (Lombardi et al. 1996). Other coupling mechanisms are also significant, involving heat transfer and surface waves (e.g. Rozenberg et al. 1998) and internal waves generated below the surface (e.g. Keeler, Bondur \& Gibson 2005).

It seems likely that the methodology outlined here can provide a useful tool for assisting in the interpretation of numerical simulations of these flows. It could also contribute to the development of appropriate models for the interfacial region in which there is a complex combination of turbulence distortion effects and other transinterfacial coupling mechanisms, both of which are likely to influence the interfacial fluxes (for a recent review, see Hasegawa \& Kasagi 2009).

This study began with an EC-funded project in 1994-1995 on atmosphere-ocean coupling. We are grateful for stimulating conversations with Professors S. Banerjee, S. Komori, G. Hewitt and K. Hasselman. J.C.R.H. is grateful for support from the NERC in the Centre for Polar Observation and Modelling at UCL, the UK Ministry of Defence, from Midi-Pyrenees Innovation at the Institut de Mé canique des Fluides de Toulouse and from Arizona State University Environmental Fluid Dynamics Program. Dr J. Clegg, the late E. Bliss and the referees helped greatly in preparing and revising the paper. 


\section{Appendix. Linear analysis of interface displacement}

In order to establish the criteria for the interface to be flat (which is assumed in the paper) we analyse small fluctuations at $z=0$, for different dynamical conditions (with or without buoyancy forces), following Carruthers \& Hunt (1986) and Fernando \& Hunt (1997). As in figure 1 , we assume high $R e$ and thin viscous layers (i.e. $\delta_{+}, \delta_{-} \ll L$ ) so that the normal velocities and pressure fluctuations are continuous, i.e.

$$
\begin{gathered}
w_{+}\left(z=\delta_{+}\right)=w_{-}\left(z=-\delta_{-}\right), \\
p_{+}\left(z=\delta_{+}\right)=p_{-}\left(z=-\delta_{-}\right),
\end{gathered}
$$

from which, taking the horizontal gradient denoted $\nabla_{h}$,

$$
\nabla_{h}^{2} p_{+}\left(z=\delta_{+}\right)=\nabla_{h}^{2} p_{-}\left(z=-\delta_{-}\right) .
$$

From the horizontal divergence of the linearized momentum equation (with $g=0$ ) we have

$$
\frac{\partial}{\partial t}\left(\rho_{+} \frac{\partial w_{+}}{\partial z}\right)=\frac{\partial}{\partial t}\left(\rho_{-} \frac{\partial w_{-}}{\partial z}\right),
$$

where

$$
\rho_{+} \frac{\partial w_{+}}{\partial z}=\rho_{-} \frac{\partial w_{-}}{\partial z} .
$$

First consider regime (i) of an interface without buoyancy forces, between a very dense fluid below a light fluid, i.e.

$$
\rho_{+} / \rho_{-} \ll 1 .
$$

The modal solution in $S_{-}$is written as a sum of the homogeneous turbulence far from the interface plus a part that decays away from the interface, as in $\S 4.1 .1$, $w_{-}=\widehat{w}_{-}^{(S)} \mathrm{e}^{-k_{12} z}+\widehat{w}_{-}^{(H)} \mathrm{e}^{\mathrm{i} k_{3} z}$. To satisfy (A $\left.3 b\right)$ and (A 4)

$$
\partial w_{-} / \partial z=-\widehat{w}_{-}^{(S)} k_{12} \mathrm{e}^{-k_{12} z}+\mathrm{i} k_{3} \widehat{w}_{-}^{(H)} \mathrm{e}^{\mathrm{i} k_{3} z}=0
$$

Thence at $z=0$,

$$
\widehat{w}_{-}^{(H)}=\left(\mathrm{i} k_{3} / k_{12}\right) \widehat{w}_{-}^{(H)}
$$

so that on taking an ensemble average

$$
\overline{w_{-}^{2}}=\left(\frac{k_{3}^{2}}{k_{12}^{2}}+1\right) w_{H}^{2}>w_{H}^{2} .
$$

This is consistent with the analysis of Eames \& Hunt (1997), showing how large fluctuations occur on the edges of dense fluid in turbulent motion.

However, if the upper layer is significantly denser than the lower layer, i.e. $\rho_{+} \gg \rho_{-}$, and there are no buoyancy forces, i.e. $g=0$, then in $S_{+}, \hat{w}_{+}=\hat{w}_{i} \mathrm{e}^{-k_{12 z} z}$, where $\hat{w}_{+}=\hat{w}_{i}$ at $z=0$, so that $\partial w_{+} / \partial z \sim\left(\rho_{-} / \rho_{+}\right)\left(\hat{w}_{H} / L\right) \ll 1$ at $z=0$. Thence

$$
\frac{\overline{w_{i}^{2}}}{\overline{w_{H}^{2}}} \sim\left(\frac{\rho_{-}}{\rho_{+}}\right)^{2} \ll 1 .
$$

In this case there are no motions on the interface which remains flat. In the presence of buoyancy forces, if the displacement is $\zeta$, the Bernoulli equation for the two layers 
can be combined. Then

$$
p_{-}-p_{+}=g\left(\rho_{-}-\rho_{+}\right) \zeta-\left[\rho_{-} \frac{\partial \phi_{-}}{\partial t}-\rho_{+} \frac{\partial \phi_{+}}{\partial t}\right] .
$$

Since $\partial \zeta / \partial t=w$, operating with $(\partial / \partial t) \nabla_{H}^{2}$ gives (for the linearized analysis)

$$
0=g \Delta \rho \nabla_{H}^{2} w_{I}-\frac{\partial^{2}}{\partial t^{2}}\left[\rho_{-} \frac{\partial w_{-}}{\partial z}-\rho_{+} \frac{\partial w_{+}}{\partial z}\right] .
$$

Thus if the buoyancy forces are large relative to the inertial forces, $F_{I}^{-1}=\left(g^{1} T_{L}^{2}\right) / L \gg 1$ where $T_{L}\left(\sim L / u_{0}\right)$ is the time scale of the fluctuations (in the upper or lower layers). Thence from $(\mathrm{A} 8 b) \nabla_{H}^{2} w=0$ at the fixed interface, $z_{i}$. However, if $F_{I}^{-1}=\left(g^{1} T_{L}^{2}\right) / L \sim 1$ the turbulence in the upper or lower layer induces waves on the interface and resonance. But where the phase speed $\left(w / k_{12}\right.$ for the given frequency and wavenumbers) is equal to the wave speed on the interface $\left((g \Delta \rho / \rho) k_{12}\right)^{1 / 2}$, wave breaking and dissipation occur (see Fernando \& Hunt 1997; Fedorov \& Mellville 1998).

\section{REFERENCES}

Belcher, S. E. \& Hunt, J. C. R. 1998 Turbulent air flow over hills and waves. Annu. Rev. Fluid Mech. 30, 507-538.

Brocchini, M. \& Peregrine, D. H. 2001 The dynamics of strong turbulence at free surface. Part 2. Free-surface boundary conditions. J. Fluid Mech. 449, 255-290.

Brutsaert, W. \& Jirka, G. H. (Ed.) 1984 Gas Transfer at Liquid Surfaces. Reidel.

CAlmet, I. \& Magnaudet, J. 2003 Statistical structure of high Reynolds number turbulence close to the free surface of an open channel flow. J. Fluid Mech. 474, 355-378.

Carruthers, D. \& Hunt, J. C. R. 1986 Velocity fluctuations near an interface between a turbulent region and a stably stratified layer. J. Fluid Mech. 156, 475-501.

Csanady, G. T. 1997 The 'slip law' of the free surface. J. Oceanogr. 53, 67-80.

DANCKwerts, P. V. 1951 Significance of liquid-film coefficients in gas absorption. Indust. Engng Chem. 43 (6), 1460-1467.

EAmes, I. \& HunT, J. C. R. 1997 Inviscid flow around bodies moving in weak density gradients without buoyancy effects. J. Fluid Mech. 353, 331-355.

Fedorov, A. V. \& Melville, W. K. 1988 Nonlinear gravity-capillary waves with forcing and dissipation. J. Fluid Mech. 354, 1-42.

Fernando, H. J. S. \& Hunt, J. C. R. 1997 Turbulence, waves and mixing at shear-free density interfaces. Part 1. A theoretical model. J. Fluid Mech. 347, 197-234.

Fulgosi, M., Lakeland, D., Banerjee, S. \& De Angelis, V. 2003 Direct numerical simulation of turbulence in a sheared air-water flow with a deformable interface. J. Fluid Mech. 482, 319-345.

GiLl, A. E. 1993 Atmosphere-Ocean Dynamics. Academic.

Guezennec, Y., Stretch, D. D. \& Kim, J. 1990 The structure of turbulent channel flow with passive scalar transport. In Studying Turbulence Using Numerical Simulation Databases, 3: Proceedings of the Summer Program 1990 (ed. P. Moin, W. C. Reynolds \& J. Kim), pp. 127-138. Center for Turbulence Research, Stanford University.

Hasegawa, Y. \& Kasagi, N. 2009 Hybrid DNS/LES of high Schmidt number mass transfer across turbulent air-water interface. Intl J. Heat Mass Transfer 52, 1012-1022.

Hunt, J. C. R. 1984 Turbulence structure in thermal convection and shear-free boundary layers. J. Fluid Mech. 138, 161-184.

Hunt, J. C. R. \& Carruthers, D. J. 1990 Rapid distortion theory and the problems of turbulence J. Fluid Mech. 212, 497-532.

Hunt, J. C. R. \& Durbin, P. A. 1999 Perturbed vortical layers and shear sheltering. Fluid Dyn. Res. 24, 375-404. 
Hunt, J. C. R. \& Graham, J. M. R. 1978 Free-stream turbulence near plane boundaries. J. Fluid Mech. 84, 209-235.

Hunt, J. C. R., Vrieling, A. J., Nieunstadt, F. T. M. \& Fernando, H. J. S. 2003 The influence of the thermal diffusivity of the lower boundary on eddy motion in convection. J. Fluid Mech. 491, 183-205.

Keeler, R. N., Bondur, V. G. \& Gibson, C. H. 2005 Optical satellite imagery detection of internal wave effects from a submerged turbulent outfall in the stratified ocean. Geophys. Res. Lett. 32, L12610.

Kim, J., Moin, P. \& Moser, R. 1987 Turbulence statistics in fully developed channel flow at low Reynolds number. J. Fluid Mech. 177, 133-166.

Komori, S., NAgaOSA, R. \& Murakami, Y. 1993 Turbulence structure and mass transfer across a sheared air-water interface in wind-driven turbulence. J. Fluid Mech. 249, 161183.

LeE, H., Moin, P. \& Kim, J. 1997 Direct numerical simulation of turbulent flow over a backwardfacing step. J. Fluid Mech. 330, 349-374.

LeE, M. J. \& Hunt, J. C. R. 1988 The structure of sheared turbulence near a plane boundary. In Studying Turbulence Using Numerical Simulation Databases, 2. Proceedings of the Summer Program 1988 (ed. P. Moin, W. C. Reynolds \& J. Kim), pp. 221-242. Center for Turbulence Research, Stanford University.

Lin, M.-Y., Moeng, C.-H., Tsai, W., Sullivan, P. P. \& Belcher, S. E. 2008 Direct simulation of wind wave generation processes. J. Fluid Mech. 616, 1-30.

Lombardi, P., De Angelis, V. \& BannerJee, S. 1996 Direct numerical simulations of near-interface turbulence in coupled gas-liquid flows. Phys. Fluids 8 (6), 1643-1665.

Magnaudet, J. 2003 High-Reynolds-number turbulence in a shear-free boundary layer: revisiting the Hunt-Graham theory. J. Fluid Mech. 484, 167-196.

Mann, J. 1994 The spatial structure of surface-layer turbulence. J. Fluid Mech. 273, 141-168.

Nagata, K., Wong, H., Hunt, J. C. R., Sajuadi, S. G. \& Davidson, P. A. 2006 Weak mean flows induced by anisotropic turbulence impinging onto planar and undulating surfaces. J. Fluid Mech. 556, 329-360.

Phillips, O. M. 1955 The irrotational motion outside a free turbulent boundary. Proc. Camb. Phil. Soc. 51, 220-229.

Rozenberg, A., Matusov, P. \& Mellvile, W. K. 1998 Polarized microwave setting by surface water waves and turbulence. IEEE Trans. Geosci. Remote Sens. 34 (6), 1331-1342.

TAKAGAKI, N. \& KoMORI, S. 2007 Effects of rainfall on mass transfer across the air-water interface. J. Geophys. Res. 112, C06006.

Teixeira, M. A. C. \& Belcher, S. E. 2002 On the distortion of turbulence by a progressive surface wave. J. Fluid Mech. 458, 229-267.

Teixeira, M. A. C. \& Belcher S. E. 2006 Initiation of surface waves by turbulence. Dyn. Atmos. Oceans 41, 1-27.

Thompson, S. M. \& Turner, J. S. 1975 Mixing across an interface due to turbulence generated by an oscillating grid. J. Fluid Mech. 67, 349-368.

Thorpe, S. A. 2004 Langmuir circulation. Annu. Rev. Fluid Mech. 36, 55-79.

Townsend, A. A. 1976 The Structure of Turbulent Shear Flows. Cambridge University Press.

TSAI, W. T. 1998 A numerical study of the evolution and structure of a turbulent shear layer under a free surface. J. Fluid Mech. 354, 239-274.

Wu, P. C. \& Fernando, H. J. S. 1999 An analyses of turbulent motions in and around a differential forced density interface. Theor. Comput. Fluid Dyn. 13, 129-141.

ZAKI, T. A. \& SAHA, S. 2009 On shear-sheltering and the structure of vortical modes in single and two-fluid boundary layers. J. Fluid Mech. 626, 111-148.

Zilitinkevich, S. S., Hunt, J. C. R., Esau, I. N., Grachev, A. A., Lalas, D. P., Akylas, E., Tombrou, M., Fairall, C. W., Fernando, H. J. S., Baklanov, A. \& Joffre, S. M. 2006 The influence of large convective eddies on the surface layer turbulence. Q. J. R. Meteorol. Soc. 132, 1423-1456. 